CLNS 92/1147, SPhT-92-054

\title{
The Quantum Double in Integrable Quantum Field Theory
}

\author{
DENIS BERNARD \\ Service de Physique Théorique 1 \\ CEN-Saclay \\ 91191 Gif sur Yvette, France. \\ ANDRÉ LECLAIR \\ Newman Laboratory \\ Cornell University \\ Ithaca, NY 14853
}

\begin{abstract}
Various aspects of recent works on affine quantum group symmetry of integrable $2 \mathrm{~d}$ quantum field theory are reviewed and further clarified. A geometrical meaning is given to the quantum double, and other properties of quantum groups. Multiplicative presentations of the Yangian double are analyzed.
\end{abstract}

$04 / 92$

1 Laboratoire de la Direction des Sciences de la Matière du Commisariat à l'Energie Atomique. 


\section{Introduction.}

Over the past few years it was realized that integrable quantum field theories in 2 spacetime dimensions are characterized by infinite dimensional Hopf algebra symmetries, i.e. affine quantum groups. These symmetries are generated by non-local quantum conserved currents which may be constructed explicitly. Previous investigations of integrable quantum field theory were based largely on the quantum inverse scattering method [1] [2], which deals with local integrals of motion in involution. The latter are useful for diagonalizing the Hamiltonian in an algebraic Bethe ansatz scheme. On the other hand, the non-local conserved charges generate non-abelian symmetries, and their power resides in the rich algebraic structures involved. Being genuine symmetries of the field theory, they constrain the S-matrices, form factors, and perhaps even the correlation functions. There is growing evidence that the affine quantum group symmetries completely characterize the models, though much remains to be understood towards the realization of this idea.

Let us review some previous results and the field theories we are implicitly dealing with. Conserved currents which generate the quantum affine algebra $\widehat{S L}_{q}$ in the sineGordon theory were constructed in [3] [4] [5]. This construction generalizes to the $\widehat{G}$-affine

Toda theories with $\widehat{G}_{q}$ symmetry[ [4]. At special values of the coupling where these quantum field theories have ordinary Lie group $G$ invariance, the quantum affine symmetry becomes the G-Yangian symmetry. The Yangian conserved currents were constructed in [6] [7]. Generic perturbations of rational conformal field theories are also characterized by nonlocal symmetries which can be obtained as restrictions of the above ones (see [8] [3] [5]). The affine quantum group invariance fixes the S-matrices up to overall scalar factors, which in turn can be fixed from crossing symmetry, unitarity, and the minimality assumption. These quantum group invariant S-matrices automatically satisfy the Yang-Baxter equation. Thus one sees that bootstrap principles (see e.g. [9] ) are replaced by, and actually a consequence of, the quantum affine symmetries. The above properties of the S-matrix are the simplest example of this idea.

In the paper [10] the quantum double of the symmetry was introduced in connection with the construction of covariant field multiplets and their form factors. A lattice construction of field multiplets was given in [11]. Smirnov showed that the basic axioms of the form factor bootstrap program may be viewed as a consequence of the affine quantum group symmetries 12]. In particular, using ideas introduced by Frenkel and Reshetikhin [13], he showed how the periodicity properties of form factors correspond to the deformed KnizhnikZamolodchikov equations introduced in [13]. Interesting new results in this direction were 
obtained in [14]. The form factor axioms were originally derived from bootstrap ideas [15], so again we see that the bootstrap can be replaced with constructions based on symmetries.

The purpose of the present work is to clarify some of the above results, and to modestly extend them. By introducing the general geometrical features of non-local conserved currents, we provide a field theoretic meaning to all of the algebraic properties of quantum groups. Some aspects of these results previously appeared in [7] [4] [10] [11] and in a conformal field theory context in [16] [17]. In particular we give a geometrical meaning to the quantum double. As will be explained, the dual charges of the double are on a completely different footing from the original non-local charges, and actually do not correspond to conserved currents in the usual sense. Nevertheless they are essential for characterizing completely the S-matrix, including scalar factors, and the braiding of non-local field multiplets.

The primary example developed in detail in this paper is the $S L(2)$ Yangian, though the results generalize to Yangians for other groups and to the $\widehat{G}_{q}$ case as well. The field theory we will be implicitly dealing with may be formulated in a variety of ways. As mentioned above, it is equivalent to the sine-Gordon model at the $S L(2)$ invariant point, i.e. at the value of the coupling $\beta$ where the $\cos (\beta \Phi)$ potential is marginal (conformal dimension $(1,1)$ ). It may also be formulated as an $S L(2)$ non-abelian Thirring model, or chiral Gross-Neveu model. For purposes of our discussion, we choose to formulate it as a current-current perturbation of the level-1 Wess-Zumino-Witten model (WZW) [18]:

$$
S=S_{W Z W}+\lambda \int d^{2} x \sum_{a} J_{\mu}^{a} J_{\mu}^{a}
$$

where $J_{\mu}^{a}(x)$ are the local $S L(2)$ currents. The equivalence with the previously mentioned models can be established using bosonization. (See e.g. [19] [4].) The beta function for $\lambda$ is non-zero, and for the appropriate sign of $\lambda$, this defines a massive field theory. The spectrum is known to consist of an $S L(2)$ doublet of solitons. The S-matrix was conjectured in [20].

The model (1.1) is characterized by the Yangian double symmetry. This algebra is a deformation of the full $S L(2)$ affine Lie algebra [10]. Though the WZW theory is characterized by $S L(2)$ affine Lie algebra symmetry, we emphasize that the Yangian double symmetry is not to be thought of as a deformation of the affine symmetry of the conformal WZW model. In other words, formally setting the deformation parameter to zero in the Yangian charges does not recover the affine Lie algebra charges of the WZW model; i.e. the 
deformation parameter has nothing to do with $\lambda$. Rather the Yangian symmetry actually exists in the conformal WZW model itself (actually, two copies of it), and is independent of the usual affine Lie symmetry. Though in the context of the WZW model itself this Yangian symmetry has not been put to much use, its role is significant in the perturbed massive theory since it continues to be conserved under perturbation. Its role is thus to organize the Hilbert space of the WZW model in such a way that this structure is preserved under perturbation.

In this paper we will also give a different presentation of the Yangian double than the one studied in [10]. This is the so-called multiplicative presentation, which was defined for the Yangian in [21], and for quantum affine algebras in [22]. This presentation has certain advantages in that the relations are finite in this basis. 


\section{General Features of Quantum Group Symmetry in 2d QFT.}

In this section we will provide a quantum field theoretic meaning for all the properties that characterize a quantum group. In this context, a quantum group refers to an arbitrary quasitriangular Hopf algebra $\mathcal{A}$. A Hopf algebra is equipped with a multiplication map $m: \mathcal{A} \otimes \mathcal{A} \rightarrow \mathcal{A}$, comultiplication $\Delta: \mathcal{A} \rightarrow \mathcal{A} \otimes \mathcal{A}$, antipode $s: \mathcal{A} \rightarrow \mathcal{A}$ and counit $\epsilon: \mathcal{A} \rightarrow C$. In addition there is the construction of the universal $R$-matrix, by means of the quantum double construction. All the definitions and properties are reviewed in the appendix A, following [21] [23] 24].

\subsection{Non-Local Currents and the Origin of the Comultiplication.}

We suppose we are dealing with some 2d quantum field theory (QFT) which possesses some conserved currents $J_{a}^{\mu}(x)$ and associated charges $Q_{a}$ :

$$
\partial_{\mu} J_{a}^{\mu}(x)=0 \quad ; \quad Q_{a}=\int_{-\infty}^{+\infty} d x J_{a}^{t}(x)
$$

The currents $J_{a}^{\mu}(x)$ are allowed to be non-local, and consequently to have non-trivial braiding relations among themselves and with the other fields. Non-local fields can be described geometrically by attaching a string from $-\infty$ to the space-time location of the fields, where the exact shape of the string is irrelevant. This string is completely analogous to the disorder line that defines disorder fields. For the non-local currents in the specific models we are interested in, the string is manifest and analogous to the Mandelstam string for the soliton fields. When defining the non-local currents, one must introduce a pointsplitting. This is quite natural when one realizes that the non-local currents that have been explicitly constructed are the product of a non-local operator (disorder field) and a local one (spin field). So, we represent the non-local currents $J_{a}^{\mu}(x)$ as in fig.1.

Fig.1 
Let $\Phi_{i}(x)$ denote a set of fields, possibly non-local. The braiding of the non-local currents $J_{a}^{\mu}(x)$ with these fields can be described by the equation:

$$
J_{a}^{\mu}(x, t) \Phi_{i}(y, t)=\Theta_{a i}^{b j} \Phi_{j}(y, t) J_{b}^{\mu}(x, t) \quad ; x>y
$$

The above equation is implicitly time-ordered to the left. We express the braiding in (2.2) as an operator, as follows:

$$
J_{a}^{\mu}(x, t) \Phi_{i}(y, t)=\widehat{\Theta}_{a}^{b}\left(\Phi_{i}(y, t)\right) J_{b}^{\mu}(x, t) \quad ; x>y
$$

where $\widehat{\Theta}_{a}^{b}\left(\Phi_{i}(y, t)\right)=\Theta_{a i}^{b j} \Phi_{j}(y, t)$. The action of the operator $\widehat{\Theta}_{b}^{a}$ is therefore represented graphically as in fig.2.

Fig.2.

The action of a charge on a field is defined by the expression:

$$
\widehat{Q}_{a}\left(\Phi_{i}(y)\right)=\oint_{\gamma(y)} d z^{\nu} \epsilon_{\nu \mu} J_{a}^{\mu}(z) \Phi_{i}(y)
$$

where the contour $\gamma(y)$ starts and ends at $-\infty$ and surrounds the point $y$. Due to the conservation law for the currents, the exact shape of the contour $\gamma(y)$ is immaterial up to topological obstructions. The above action of a charge on a field can be expressed as a braided commutator by decomposing $\gamma(y)$ into the contours as in fig.3.

Fig.3 
Therefore,

$$
\widehat{Q}_{a}\left(\Phi_{i}(y)\right)=Q_{a} \Phi_{i}(y)-\widehat{\Theta}_{a}^{b}\left(\Phi_{i}(y)\right) Q_{b}
$$

In addition to defining the action of $\widehat{\Theta}_{a}^{b}$ on a field as above, we define the operator $\Theta_{a}^{b}$ itself as a string from $-\infty$ to $+\infty$. We will denote by $\mathcal{A}$ the algebra generated by the $Q_{a}$ and $\Theta_{a}^{b}$. The braided commutator (2.5) may equal a new operator if one can use the operator product expansion and close the contour on the right hand side of (2.4).

Let the braiding of the currents with themselves be given by:

$$
\begin{aligned}
J_{a}^{\mu}(x) J_{b}^{\nu}(y) & =\widehat{\Theta}_{a}^{c}\left(J_{b}^{\nu}(y)\right) J_{c}^{\mu}(x) \\
& =R_{a b}^{c d}\left(J_{d}^{\nu}(y)\right) J_{c}^{\mu}(x) ; x>y
\end{aligned}
$$

Then the integrated version of (2.5) for $\Phi_{j}(x)=J_{a}^{\mu}(x)$ is:

$$
\widehat{Q}_{a}\left(Q_{b}\right)=Q_{a} Q_{b}-R_{a b}^{c d} Q_{d} Q_{c}
$$

The comultiplication arises when one considers the action of the operators $\widehat{Q}_{a}$ and $\widehat{\Theta}_{a}^{b}$ on a product of several fields. Consider for simplicity the action on two fields:

$$
\widehat{Q}_{a}\left(\Phi_{i}\left(y_{1}\right) \Phi_{j}\left(y_{2}\right)\right)=\oint_{\gamma\left(y_{1} ; y_{2}\right)} d z^{\nu} \epsilon_{\nu \mu} J_{a}^{\mu}(z) \Phi_{i}\left(y_{1}\right) \Phi_{j}\left(y_{2}\right)
$$

where the contour $\gamma\left(y_{1} ; y_{2}\right)$ surrounds the two points $y_{1}, y_{2}$. Decomposing it into the sum of two contours surrounding respectively the points $y_{1}$ and $y_{2}$ (see figure 4 ), one obtains:

$$
\widehat{Q}_{a}\left(\Phi_{i}\left(y_{1}\right) \Phi_{j}\left(y_{2}\right)\right)=\widehat{Q}_{a}\left(\Phi_{i}\left(y_{1}\right)\right) \Phi_{j}\left(y_{2}\right)+\widehat{\Theta}_{a}^{b}\left(\Phi_{i}\left(y_{1}\right)\right) \widehat{Q}_{b}\left(\Phi_{j}\left(y_{2}\right)\right)
$$

Fig.4

Therefore,

$$
\Delta\left(\widehat{Q}_{a}\right)=\widehat{Q}_{a} \otimes 1+\widehat{\Theta}_{a}^{b} \otimes \widehat{Q}_{b}
$$

Similarly, one also obtains,

$$
\Delta\left(\widehat{\Theta}_{a}^{b}\right)=\widehat{\Theta}_{a}^{c} \otimes \widehat{\Theta}_{c}^{b}
$$




\subsection{Euclidean Rotations and the Origin of the Antipode and Counit.}

Define $R_{\pi}\left(R_{-\pi}\right)$ to be the Euclidean rotation in the $x-t$ plane by an angle of $\pi$ in the clockwise (anticlockwise) direction. For $a \in \mathcal{A}$, we define the antipode $s$ and skew-antipode $s^{\prime}$ as follows:

$$
s(a) \equiv R_{\pi}(a) \quad ; \quad s^{\prime}(a) \equiv R_{-\pi}(a) \quad ; \forall a \in \mathcal{A}
$$

By construction, one has $s s^{\prime}=s^{\prime} s=1$. The antimultiplicative property of the antipode $s(a b)=s(b) s(a)$ is a consequence of the fact that the rotations $R_{ \pm \pi}$ involve a time reversal.

Let us now derive the consequences of these definitions for the non-local charges. In order to see the difference between $R_{\pi}$ and $R_{-\pi}$ and to realize that $R_{\pi}^{2} \neq 1$, one must remember the point-splitting that we have introduced into the graphical notation of the non-local fields. The rotations $R_{\pi}$ and $R_{-\pi}$ of $J_{a}^{\mu}(x)$ have a string originating from $+\infty$ rather than $-\infty$ : for $R_{\pi}\left(J_{a}^{\mu}(x)\right)$ the string is above the points used in the point-splitting procedure while for $R_{-\pi}\left(J_{a}^{\mu}(x)\right)$ it is under. Therefore $R_{\pi} \neq R_{-\pi}$, and $R_{\pi}^{2}\left(J_{a}^{\mu}(x)\right) \neq J_{a}^{\mu}(x)$. (See figure 5.)

\section{Fig.5}

This will be used at the end of section 2.6 to derive a spin-statistics relation.

For the operators $\Theta_{a}^{b}$, the rotations of $R_{ \pm \pi}$ simply change the orientation of the contour; therefore,

$$
\Theta_{a}^{c} s\left(\Theta_{c}^{b}\right)=s^{\prime}\left(\Theta_{c}^{b}\right) \Theta_{a}^{c}=\delta_{a}^{b}
$$

The rotations of the operators $Q_{a}$ are displayed in fig.6. From them one deduces the antipodes:

$$
s\left(Q_{a}\right)=-s\left(\Theta_{a}^{b}\right) Q_{b} \quad ; \quad s^{\prime}\left(Q_{a}\right)=-Q_{b} s^{\prime}\left(\Theta_{a}^{b}\right)
$$




\section{Fig.6}

The counit $\epsilon$ of the operators $Q_{a}$ and $\Theta_{a}^{b}$ is defined to be the one-dimensional vacuum representation of these operators in the QFT. Let $I$ denote the unit operator in the QFT. The counit $\epsilon$ may then be defined by the formula:

$$
\epsilon(a)=\langle 0|a| 0\rangle \quad \forall a \in \mathcal{A},
$$

or equivalently

$$
\epsilon(a)=\widehat{a}(I),
$$

where $I$ is inserted at an arbitrary point $y$. Indeed, it is evident from (A.5i) that the counits form a one-dimensional representation of $\mathcal{A}$. By construction, the counit $\epsilon$ satisfies:

$$
(1 \otimes \epsilon) \Delta(a)=(\epsilon \otimes 1) \Delta(a)=a \quad ; \quad \forall a \in \mathcal{A}
$$

Indeed, let $\Phi(x)$ be any quantum field, then we have:

$$
(1 \otimes \epsilon) \Delta(a)(\Phi(x))=\widehat{a}(\Phi(x) I)=\widehat{a}(\Phi(x))
$$

The relation (2.17) is one of the defining properties of a Hopf algebra. Let us now derive the exact values of the counits. In eq. (2.15), for the action of $\widehat{Q}_{a}$, the contour surrounding $y$ may be shrunk to zero, therefore,

$$
\epsilon\left(Q_{a}\right)=0
$$

On the other hand, for the action of $\widehat{\Theta}_{a}^{b}$, the contour in (2.16) can be deformed to $-\infty$ to yield :

$$
\epsilon\left(\Theta_{a}^{b}\right)=\delta_{a}^{b}
$$


Finally, as a consequence of eqs. (2.13) and (2.14) and of the values of the counit, one verifies that the antipode satisfies:

$$
m(1 \otimes s) \Delta(a)=m(s \otimes 1) \Delta(a)=\epsilon(a) \quad \forall a \in \mathcal{A}
$$

where $m$ denotes the multiplication in $\mathcal{A}$. Eq. (2.21) is also one of the compatibility relations defining a Hopf algebra.

The above properties define a Hopf algebra $\mathcal{A}$, generated by the $Q_{a}$ and $\Theta_{a}^{b}$. One sees that the specific form of the various operations, e.g. the comultiplication, is a consequence of the fact that $Q_{a}$ is the integral of a conserved current. In the appendix the basic properties of a Hopf algebra $\mathcal{A}$ were described using a linear basis $\left\{e_{a}\right\}$. There, the various operations were formulated in terms of structure constants, i.e.:

$$
\begin{aligned}
e_{a} e_{b} & =m_{a b}^{c} e_{c} \\
\Delta\left(e_{a}\right) & =\mu_{a}^{b c} e_{b} \otimes e_{c} \\
s\left(e_{a}\right) & =s_{a}^{b} e_{b} \\
\epsilon\left(e_{a}\right) & =\epsilon_{a}
\end{aligned}
$$

As it turns out, there always exists a change of basis to what we call the current basis, where all the field theoretic properties described above are valid. From the general basis $\left\{e_{a}\right\}$ define the generators of the current basis as follows,

$$
Q_{a}=e_{a}-\epsilon_{a} \quad ; \quad \Theta_{a}^{b}=\mu_{a}^{c b} e_{c}
$$

Using the properties of the generators $e_{a}$ in the appendix, one verifies the relations (2.10) (2.11) (without the hat) and (2.13) (2.14) (2.19) (2.20). We will henceforth use the basis $\left\{e_{a}\right\}$ and $\left\{Q_{a}, \Theta_{a}^{b}\right\}$ interchangeably for the algebra $\mathcal{A}$.

The information about the multiplication maps for $\mathcal{A}$ is contained in the relation (2.7). For reasons that will become apparent in the next subsection, we make this connection by introducing an adjoint action of the algebra $\mathcal{A}$ on itself. For $g \in \mathcal{A}$, express $\Delta(g)$ as

$$
\Delta(g)=\sum_{i} g_{i} \otimes g^{i} \quad ; \quad g_{i}, g^{i} \in \mathcal{A}
$$

and define the adjoint action of $g$ on $h$ as:

$$
\operatorname{ad}_{g}(h)=\sum_{i} g_{i} h s\left(g^{i}\right)
$$


This adjoint action enjoys the property, for $g_{1}, g_{2} \in \mathcal{A}$,

$$
a d_{g_{1}}\left(a d_{g_{2}}(h)\right)=a d_{g_{1} g_{2}}(h), \quad h \in \mathcal{A}
$$

which is a consequence of the homomorphism property of the comultiplication and of the anti-multiplicative property of the antipode. In particular, one finds

$$
a d_{e_{a}}\left(e_{b}\right)=\tilde{f}_{a b}^{c} e_{c}
$$

where

$$
\tilde{f}_{a b}^{c}=\mu_{a}^{i j} s_{j}^{l} m_{i b}^{k} m_{k l}^{c}
$$

In the current basis, one finds,

$$
\begin{aligned}
& a d_{\Theta_{a}^{b}}\left(Q_{c}\right)=R_{a c}^{b d} Q_{d} \\
& a d_{Q_{a}}\left(Q_{b}\right)=Q_{a} Q_{b}-R_{a b}^{c d} Q_{d} Q_{c}=f_{a b}^{c} Q_{c}
\end{aligned}
$$

where

$$
\begin{aligned}
R_{a c}^{b d} & =\mu_{a}^{i b} \tilde{f}_{i c}^{d}=\mu_{a}^{i b} \mu_{i}^{j k} s_{k}^{l} m_{j b}^{n} m_{k n}^{d} \\
f_{a b}^{c} & =\widetilde{f}_{a b}^{c}-\epsilon_{a} \delta_{b}^{c}
\end{aligned}
$$

One may clearly formulate all the general Hopf algebra properties in the current basis. It is clear that (2.29) contains the information of the multiplication maps. We have already explained how the 1.h.s. of (2.29) arises as a braided commutator. As explained above, the r.h.s. of (2.29) arises when one can use the operator product expansion to compute the action of the charges on the currents.

\subsection{Adjoint Action and Field Representations.}

In the situation where one is dealing with ordinary Lie algebra symmetry in QFT generated by local currents (which just corresponds to $\Theta_{a}^{b}=\delta_{a}^{b}$ above) one finds that fields can be organized into irreducible representations of the Lie algebra. The action of a conserved charge an a field is simply the commutator of the charge with the field. Starting from a highest weight field, one can complete the representation by acting with appropriate raising or lowering operators. That the fields related via this action necessarily form a representation of the Lie algebra is ensured by the Jacobi identity.

The above notions may be generalized to the situation of an arbitrary Hopf algebra

symmetry. Several different definitions of the adjoint action of a charge on a field are 
possible; for the case of Lie algebra symmetry they are equivalent. Let $g \in \mathcal{A}$, with $\Delta(g)=\sum g_{i} \otimes g^{i}$. We define two different adjoint actions of $g$ on a field $\Phi(x)$ as follows:

$$
\begin{aligned}
& \operatorname{ad}_{g}(\Phi(x))=\sum_{i} g_{i} \Phi(x) s\left(g^{i}\right) \\
& a d_{g}^{\prime}(\Phi(x))=\sum_{i} g^{i} \Phi(x) s^{\prime}\left(g_{i}\right)
\end{aligned}
$$

Using the Hopf algebra properties outlined in the appendix, one shows that the adjoint actions $a d$ and $a d^{\prime}$ enjoy the following properties:

$$
\begin{aligned}
& a d_{g_{1}}\left(a d_{g_{2}}(\Phi(x))\right)=a d_{g_{1} g_{2}}(\Phi(x)) \\
& a d_{g_{1}}^{\prime}\left(a d_{g_{2}}^{\prime}(\Phi(x))\right)=a d_{g_{1} g_{2}}^{\prime}(\Phi(x))
\end{aligned}
$$

Furthermore,

$$
\begin{aligned}
& a d_{g}\left(\Phi_{1}\left(x_{1}\right) \Phi_{2}\left(x_{2}\right)\right)=\sum_{i} a d_{g_{i}}\left(\Phi_{1}\left(x_{1}\right) a d_{g^{i}}\left(\Phi_{2}\left(x_{2}\right)\right)\right. \\
& a d_{g}^{\prime}\left(\Phi_{1}\left(x_{1}\right) \Phi_{2}\left(x_{2}\right)\right)=\sum_{i} a d_{g^{i}}^{\prime}\left(\Phi_{1}\left(x_{1}\right) a d_{g_{i}}^{\prime}\left(\Phi_{2}\left(x_{2}\right)\right)\right.
\end{aligned}
$$

The adjoint actions (2.32) are "left" actions and one also has the possibility of defining two "right" actions:

$$
\begin{aligned}
a d_{g}^{R}(\Phi(x)) & =\sum_{i} s\left(g_{i}\right) \Phi(x) g^{i} \\
a d_{g}^{\prime} R(\Phi(x)) & =\sum_{i} s^{\prime}\left(g^{i}\right) \Phi(x) g_{i}
\end{aligned}
$$

These actions possess the analogous properties:

$$
\begin{aligned}
a d_{g_{1}}^{R}\left(a d_{g_{2}}^{R}(\Phi(x))\right) & =a d_{g_{2} g_{1}}^{R}(\Phi(x)) \\
a d_{g_{1}}^{\prime R}\left(a d_{g_{2}}^{\prime R}(\Phi(x))\right) & =a d_{g_{2} g_{1}}^{\prime R}(\Phi(x))
\end{aligned}
$$

Henceforth, we will only be using the left actions.

For the generators $Q_{a}$ and $\Theta_{a}^{b}$, the adjoint actions (2.31) read:

$$
\begin{aligned}
& a d_{\Theta_{a}^{b}}(\Phi(x))=\Theta_{a}^{c} \Phi(x) s\left(\Theta_{c}^{b}\right) \\
& a d_{Q_{a}}(\Phi(x))=Q_{a} \Phi(x)-a d_{\Theta_{a}^{b}}(\Phi(x)) Q_{b}
\end{aligned}
$$

and

$$
\begin{aligned}
& a d_{\Theta_{a}^{b}}^{\prime}(\Phi(x))=\Theta_{c}^{b} \Phi(x) s^{\prime}\left(\Theta_{a}^{c}\right) \\
& a d_{Q_{a}}^{\prime}(\Phi(x))=\Phi(x) s^{\prime}\left(Q_{a}\right)+Q_{b} \Phi(x) s^{\prime}\left(\Theta_{a}^{b}\right)
\end{aligned}
$$


Using the graphical representations for $Q_{a}$ and $\Theta_{a}^{b}$ and their antipodes, one can formulate the equations (2.36) and (2.37). In this way it is clear that the graphical formulation of the r.h.s. of (2.36) is the same as displayed for the operators $Q_{a}$ and $\Theta_{a}^{b}$ in fig.2 and fig.3 respectively. Therefore, we have an equivalence between the formula (2.36) and the actions on fields $\widehat{Q}_{a}$ and $\widehat{\Theta}_{a}^{b}$ defined in section 2.1.:

$$
\begin{aligned}
& \operatorname{ad}_{Q_{a}}(\Phi(x))=\widehat{Q}_{a}(\Phi(x)) \\
& \operatorname{ad}_{\Theta_{a}^{b}}(\Phi(x))=\widehat{\Theta}_{a}^{b}(\Phi(x))
\end{aligned}
$$

The graphical formulation of eq. (2.37) is formulated similarly, and one finds that the contours originate at $+\infty$ rather that $-\infty$. (See figure 7 .)

\section{Fig. 7}

A definition of a highest weight field $\Phi_{\lambda}(x)$ will be given in the next section. Starting from such a highest weight field, repeated $a d$ or $a d^{\prime}$ action yields a tower of its descendent fields $\Phi_{\Lambda}$ and $\Phi_{\Lambda}^{\prime}$ :

$$
\Phi_{\lambda}(x) \longrightarrow^{a d} \Phi_{\Lambda}(x) \quad ; \quad \Phi_{\lambda}(x) \longrightarrow^{a d^{\prime}} \Phi_{\Lambda}^{\prime}(x)
$$

In general, the fields $\Phi_{\Lambda}(x)$ or $\Phi_{\Lambda}^{\prime}(x)$ will be non-local, since the charges are non-local. The fields $\Phi_{\Lambda}(x)$ and $\Phi_{\Lambda}^{\prime}(x)$ span a vector space $\Lambda$. We denote individual fields in this vector space as $\Phi_{v}(x)$ and $\Phi_{v}^{\prime}(x), v \in \Lambda$. Due to the properties (2.32), the fields $\Phi_{\Lambda}(x)$ and $\Phi_{\Lambda}^{\prime}(x)$ necessarily form a representation of $\mathcal{A}$ :

$$
\begin{aligned}
& a d_{g}\left(\Phi_{v}(x)\right)=\rho_{\Lambda}(g)_{v}^{w} \Phi_{w}(x) \equiv \Phi_{g \cdot v}(x) \\
& a d_{g}^{\prime}\left(\Phi_{v}^{\prime}(x)\right)=\rho_{\Lambda}^{\prime}(g)_{v}^{w} \Phi_{w}^{\prime}(x) \equiv \Phi_{g \cdot v}^{\prime}(x)
\end{aligned}
$$

In general, for the affine quantum groups the fields $\Phi_{\Lambda}$ will form infinite dimensional Verma module representations. 
Using the Hopf algebra properties one can show that for $g \in \mathcal{A}$ :

$$
\begin{gathered}
g \Phi(x)=a d_{g_{i}}(\Phi(x)) g^{i} \\
g \Phi(x)=a d_{g^{i}}^{\prime}(\Phi(x)) g_{i} \\
\Phi(x) s(g)=s\left(g_{i}\right) a d_{g^{i}}(\Phi(x)) \\
\Phi(x) s^{\prime}(g)=s^{\prime}\left(g^{i}\right) a d_{g_{i}}^{\prime}(\Phi(x))
\end{gathered}
$$

where $g_{i}$ and $g^{i}$ are defined in eq. (2.24). By comparing the graphical formulation for $a d_{g}(\Phi(x))$ and $a d_{g}^{\prime}(\Phi(x))$ when $g=Q_{a}$ or $\Theta_{a}^{b}$, one sees that $\Phi_{\Lambda}(x)$ and $\Phi_{\Lambda}^{\prime}(x)$ are simply related by a rotation:

$$
\Phi_{\Lambda}^{\prime}(0)=R_{-\pi}\left(\Phi_{\Lambda}(0)\right)
$$

Indeed, eq. (2.42) ensures that if $\Phi_{\Lambda}(x)$ is ad-covariant, then $\Phi_{\Lambda}^{\prime}(x)$ is $a d^{\prime}$-covariant. The proof is straightforward:

$$
\begin{aligned}
R_{-\pi}\left(a d_{g}\left(\Phi_{v}(0)\right)\right) & =R_{-\pi}\left(g_{i} \Phi_{v}(0) s\left(g^{i}\right)\right) \\
& =s^{\prime} s\left(g^{i}\right) R_{-\pi}\left(\Phi_{v}(0)\right) s^{\prime}\left(g_{i}\right) \\
& =a d_{g}^{\prime}\left(\Phi_{v}^{\prime}(0)\right)
\end{aligned}
$$

In the second step we have used the fact that $R_{-\pi}$ involves a time reversal, and also the relation $s s^{\prime}=1$.

\subsection{The Geometrical Meaning of the Quantum Double.}

As before, we let $\left\{e_{a}\right\}$ denote a general linear basis for $\mathcal{A}$. The charges $e_{a}$ act on a field via the adjoint actions defined above:

$$
\begin{aligned}
& a d_{e_{a}}\left(\Phi_{v}(x)\right)=\mu_{a}^{b c} e_{b} \Phi_{v}(x) s\left(e_{c}\right) \equiv \Phi_{e_{a} \cdot v}(x) \\
& a d_{e_{a}}^{\prime}\left(\Phi_{v}^{\prime}(x)\right)=\mu_{a}^{b c} e_{c} \Phi_{v}^{\prime}(x) s^{\prime}\left(e_{b}\right) \equiv \Phi_{e_{a} \cdot v}^{\prime}(x)
\end{aligned}
$$

In seeking an operator formulation of the rotations $R_{ \pm \pi}$, one is led to define a different dual Hopf algebra $\mathcal{A}^{*}$ whose properties can be derived from its operational definition. We let $\left\{e^{a}\right\}$ denote a linear basis for $\mathcal{A}^{*}$. We will give two different, but closely related definitions of the action of $e^{a}$ on fields, one being more suitable than the other, depending on which property of $e^{a}$ one is interested in. We denote the action of $e^{a}$ on a field as:

$$
e^{a}\left(\Phi_{v}(x)\right) \equiv \Phi_{e^{a} \cdot v}(x)
$$


and similarly for $e^{a}\left(\Phi_{v}^{\prime}(x)\right)$. We emphasize that we do not assume the elements $e^{a}$ can be defined in the QFT as integrals of conserved currents, the way the elements of $\mathcal{A}$ were. Rather we provide a set of 'instructions' for defining only the action of $e^{a}$ on a field, and then proceed to derive its algebraic properties.

The first definition of $e^{a}\left(\Phi_{v}(x)\right)$ is with respect to the braiding of fields:

$$
\begin{aligned}
& \Phi_{v_{1}}\left(x_{1}\right) \Phi_{v_{2}}\left(x_{2}\right)=\sum_{a} \Phi_{e_{a} \cdot v_{2}}\left(x_{2}\right) \Phi_{e^{a} \cdot v_{1}}\left(x_{1}\right) \quad ; \quad x_{1}>x_{2} \\
& \Phi_{v_{1}}^{\prime}\left(x_{1}\right) \Phi_{v_{2}}^{\prime}\left(x_{2}\right)=\sum_{a} \Phi_{e^{a} \cdot v_{2}}^{\prime}\left(x_{2}\right) \Phi_{e_{a} \cdot v_{1}}^{\prime}\left(x_{1}\right) \quad ; \quad x_{1}>x_{2}
\end{aligned}
$$

In the above braiding relations, the fields $\Phi_{v_{1}}(x)$ and $\Phi_{v_{2}}(x)$ may be part of two different representations. The algebraic relations among the elements of $\mathcal{A}$ and $\mathcal{A}^{*}$ arises when one considers the $\mathcal{A}$-covariance of (2.46). Applying $a d_{e_{a}}$ to both sides of eq. (2.46) and using eq. (2.33) one obtains:

$$
\begin{aligned}
a_{e_{a}} \text { (L.H.S.) } & =\mu_{a}^{b c} \Phi_{e_{b} \cdot v_{1}}\left(x_{1}\right) \Phi_{e_{c} \cdot v_{2}}\left(x_{2}\right) \\
& =\mu_{a}^{b c} \Phi_{e_{n} e_{c} \cdot v_{2}}\left(x_{2}\right) \Phi_{e^{n} e_{b} \cdot v_{1}}\left(x_{1}\right)
\end{aligned}
$$

whereas

$$
a d_{e_{a}} \text { (R.H.S.) }=\mu_{a}^{b c} \Phi_{e_{b} e_{n} \cdot v_{2}}\left(x_{2}\right) \Phi_{e_{c} e^{n} \cdot v_{1}}\left(x_{1}\right)
$$

Equating (2.47) and (2.48) one obtains :

$$
\mu_{a}^{b c} m_{n c}^{d} e^{n} e_{b}=\mu_{a}^{b c} m_{b n}^{d} e_{c} e^{n}
$$

The $\mathcal{A}$-covariance of the braiding relations for the $a d^{\prime}$-covariant fields is also ensured by eq.(2.49). Eq. (2.49) gives the commutation relations between the $\left\{e_{a}\right\}$ and the $\left\{e^{b}\right\}$.

The multiplication map in $\mathcal{A}^{*}$ can be derived by requiring that the algebra (2.46) is associative when one braids a product of three fields. This requirement is equivalent to the Yang-Baxter equation (A.9), which is satisfied as a consequence of (2.49) if the following additional relations are imposed:

$$
e^{a} e^{b}=\mu_{c}^{b a} e^{c}
$$

Thus, the braiding of field multiplets is given by the universal $\mathcal{R}$-matrix evaluated in the representations $\rho_{\Lambda_{1}}, \rho_{\Lambda_{2}}$ :

$$
\Phi_{\Lambda_{1}}\left(x_{1}\right) \Phi_{\Lambda_{2}}\left(x_{2}\right)=\mathcal{R}_{\rho_{\Lambda_{2}}, \rho_{\Lambda_{1}}} \Phi_{\Lambda_{2}}\left(x_{2}\right) \Phi_{\Lambda_{1}}\left(x_{1}\right) ; \quad x_{1}>x_{2}
$$


The second definition of the action of $e^{a}$ on a field involves directly the rotation $R_{ \pm \pi}$, and is conceptually more fundamental,

$$
\begin{aligned}
R_{\pi}\left(\Phi_{v}^{\prime}(0)\right) & =\Phi_{v}(0)=e^{a}\left(\Phi_{v}^{\prime}(0)\right) e_{a} \\
R_{-\pi}\left(\Phi_{v}(0)\right) & =\Phi_{v}^{\prime}(0)=e^{a}\left(\Phi_{v}(0)\right) s^{\prime}\left(e_{a}\right)
\end{aligned}
$$

One may check that the formulas (2.52) are covariant if (2.49) is satisfied. I.e. the formulas (2.52) are compatible with the covariance properties (2.44) of both field multiplets $\Phi_{v}$ and $\Phi_{v}^{\prime}$. Let us consider the first of the relations (2.52). To prove the covariance, we begin with the following relations (eq. (2.41)):

$$
\begin{aligned}
& e_{a} \Phi_{v}=\mu_{a}^{b c} \Phi_{e_{b} \cdot v} e_{c} \\
& e_{a} \Phi_{v}^{\prime}=\mu_{a}^{b c} \Phi_{e_{c} \cdot v}^{\prime} e_{b} .
\end{aligned}
$$

The latter imply

$$
e_{a} \Phi_{v}=e_{a} \Phi_{e^{n} \cdot v}^{\prime} e_{n}=\mu_{a}^{b c} m_{b n}^{d} \Phi_{e_{c} e^{n} \cdot v}^{\prime} e_{d}
$$

On the other hand, one also has,

$$
\mu_{a}^{b c} \Phi_{e_{b} \cdot v} e_{c}=\mu_{a}^{b c} \Phi_{e^{n} e_{b} \cdot v}^{\prime} e_{n} e_{c}=\mu_{a}^{b c} m_{n c}^{d} \Phi_{e^{n} e_{b} \cdot v}^{\prime} e_{d}
$$

Equating (2.54) and (2.55) one concludes that (2.52) is covariant if (2.49) is satisfied. In the same way one verifies that the second relation (2.52) is also covariant.

The definition (2.52) of $e^{a}(\Phi(x))$ allows one to derive the comultiplication $\Delta\left(e^{a}\right)$. This comultiplication arises when one considers the action of $e^{a}$ on a product of two fields. The definition (2.52) extended to such a product reads:

$$
\begin{aligned}
\Phi_{v_{1}}\left(x_{1}\right) \Phi_{v_{2}}\left(x_{2}\right) & =e^{a}\left(R_{-\pi}\left(\Phi_{v_{1}}\left(x_{1}\right) \Phi_{v_{2}}\left(x_{2}\right)\right)\right) e_{a} \\
& =e^{a}\left(\Phi_{v_{2}}^{\prime}\left(x_{2}\right) \Phi_{v_{1}}^{\prime}\left(x_{1}\right)\right) e_{a}
\end{aligned}
$$

Let $\Delta^{\prime}\left(e^{a}\right)=L_{b c}^{a} e^{c} \otimes e^{b}$. Then,

$$
\begin{aligned}
e^{a}\left(\Phi_{v_{2}}^{\prime}\left(x_{2}\right) \Phi_{v_{1}}^{\prime}\left(x_{1}\right)\right) e_{a} & =e^{a}\left(\Phi_{e^{n} \cdot v_{1}}^{\prime}\left(x_{1}\right) \Phi_{e_{n} \cdot v_{2}}^{\prime}\left(x_{2}\right)\right) e_{a} \\
& =L_{k l}^{a} \Phi_{e^{l} e^{n} \cdot v_{1}}^{\prime}\left(x_{1}\right) \Phi_{e^{k} e_{n} \cdot v_{2}}^{\prime}\left(x_{2}\right) e_{a} \\
& =L_{k l}^{a} \mu_{m}^{n l} \Phi_{e^{m} \cdot v_{1}}^{\prime}\left(x_{1}\right) \Phi_{e^{k} e_{n} \cdot v_{2}}^{\prime}\left(x_{2}\right) e_{a}
\end{aligned}
$$

On the other hand,

$$
\begin{aligned}
\Phi_{v_{1}}\left(x_{1}\right) \Phi_{v_{2}}\left(x_{2}\right) & =\Phi_{e^{k} \cdot v_{1}}^{\prime}\left(x_{1}\right) e_{k} \Phi_{e^{j} \cdot v_{2}}^{\prime}\left(x_{2}\right) e_{j} \\
& =\mu_{k}^{s r} \Phi_{e^{k} \cdot v_{1}}^{\prime}\left(x_{1}\right) \Phi_{e_{r} e^{j} \cdot v_{2}}^{\prime}\left(x_{2}\right) e_{s} e_{j} \\
& =\mu_{k}^{s r} m_{s j}^{n} \Phi_{e^{k} \cdot v_{1}}^{\prime}\left(x_{1}\right) \Phi_{e_{r} e^{j} \cdot v_{2}}^{\prime}\left(x_{2}\right) e_{n}
\end{aligned}
$$


Equating (2.57) and (2.58) requires

$$
L_{s r}^{i} \mu_{k}^{j r} e^{s} e_{j}=\mu_{k}^{s r} m_{s j}^{i} e_{r} e^{j}
$$

Comparing with eq. (2.49), one concludes :

$$
\Delta\left(e^{a}\right)=m_{b c}^{a} e^{b} \otimes e^{c} .
$$

The same comultiplication can be derived from the second relation (2.52). The antipode and counit in $\mathcal{A}^{*}$ can be fixed to be consistent with the above multiplication and comultiplication, as in the appendix: $\epsilon\left(e^{a}\right)=\epsilon^{a}, s\left(e^{a}\right)=\left(s^{\prime}\right)_{b}^{a} e^{b}$. One also has $\epsilon_{a} e^{a}=1$. The relations (2.22) and (2.49) (2.50) (2.60) define the double Hopf algebra $\mathcal{D}(\mathcal{A})$.

We are now in position to define more precisely a highest weight field. Consider highest weight fields that are defined to be annihilated by the elements of $\mathcal{A}^{*}$. Recall the counit was defined to be the one-dimensional vacuum representation and we take the same definition for $\mathcal{A}^{*}$. The statement that $e^{a}$ annihilate highest weight fields amounts to demanding that $e^{a}$ on $\Phi_{\lambda}(x)$ gives this one-dimensional representation:

$$
e^{a}\left(\Phi_{\lambda}(x)\right)=\epsilon^{a} \Phi_{\lambda}(x) \quad ; \quad e^{a}\left(\Phi_{\lambda}^{\prime}(x)\right)=\epsilon^{a} \Phi_{\lambda}^{\prime}(x)
$$

One can show using the properties in appendix A that $\epsilon^{a}$ indeed defines a one-dimensional representation of $\mathcal{A}^{*}: \epsilon^{a} \epsilon^{b}=\mu_{c}^{b a} \epsilon^{c}$. This definition is consistent with the fact that for highest weight fields $\Phi_{\lambda}$ must equal $\Phi_{\lambda}^{\prime}$; from (2.52) one has:

$$
\Phi_{\lambda}=e^{a}\left(\Phi_{\lambda}^{\prime}\right) e_{a}=\Phi_{\lambda}^{\prime} \epsilon^{a} e_{a}=\Phi_{\lambda}^{\prime}
$$

Highest weight fields defined this way are necessarily local with respect to each other; from (2.46) one has:

$$
\begin{aligned}
\Phi_{\lambda_{1}}\left(x_{1}\right) \Phi_{\lambda_{2}}\left(x_{2}\right) & =\Phi_{e_{n} \cdot \lambda_{2}}\left(x_{2}\right) \Phi_{e^{n} \cdot \lambda_{1}}\left(x_{1}\right) \\
& =\epsilon^{n} \Phi_{e_{n} \cdot \lambda_{2}}\left(x_{2}\right) \Phi_{\lambda_{1}}\left(x_{1}\right) \\
& =\Phi_{\lambda_{2}}\left(x_{2}\right) \Phi_{\lambda_{1}}\left(x_{1}\right)
\end{aligned}
$$

In order to characterize all the highest weight fields, including e.g. the soliton fields that create massive particles, one must extend the above definition of highest weight field to include non-local ones. More generally then, one can define highest weight fields to form a finite dimensional representation of some abelian subalgebra of the double. For example, 
in the case of the Yangian, the soliton fields can be taken as highest weight, transforming in the spinor representation of the $S L(2)$ subalgebra.

Finally, it is possible to establish directly the equivalence of the two above definitions of the action of $e^{a}$ on a field by actually deriving the braiding relation (2.46) from (2.52). Since $\Phi_{\Lambda}$ and $\Phi_{\Lambda}^{\prime}$ are related by a rotation $R_{\pi}$, whereas the strings that characterize the non-locality of $\Phi_{\Lambda}$ originate at $-\infty$, those for $\Phi_{\Lambda}^{\prime}$ originate at $+\infty$. Therefore, there is no obstruction to deforming the contour in the product of $\Phi_{v_{1}}\left(x_{1}\right) \Phi_{v_{2}}^{\prime}\left(x_{2}\right)$ for $x_{1}<x_{2}$; thus

$$
\Phi_{v_{1}}\left(x_{1}\right) \Phi_{v_{2}}^{\prime}\left(x_{2}\right)=\Phi_{v_{2}}^{\prime}\left(x_{2}\right) \Phi_{v_{1}}\left(x_{1}\right) \quad ; x_{1}<x_{2}
$$

Inserting (2.52) into (2.64) one has:

$$
\Phi_{v_{1}}\left(x_{1}\right) s\left(e_{a}\right) \Phi_{e^{a} \cdot v_{2}}\left(x_{2}\right)=s\left(e_{a}\right) \Phi_{e^{a} \cdot v_{2}}\left(x_{2}\right) \Phi_{v_{1}}\left(x_{1}\right)
$$

Replacing $v_{2}$ by $e^{b} \cdot v_{2}$ and using (2.50), eq. (2.65) becomes:

$$
\mu_{c}^{b a} \Phi_{v_{1}}\left(x_{1}\right) s\left(e_{a}\right) \Phi_{e^{c} \cdot v_{2}}\left(x_{2}\right)=\mu_{c}^{b a} s\left(e_{a}\right) \Phi_{e^{c} \cdot v_{2}}\left(x_{2}\right) \Phi_{v_{1}}\left(x_{1}\right)
$$

Multiplying both sides of (2.66) by $e_{b}$ and summing over $b$ one obtains (2.46).

\subsection{Adjoint Representations of the Double and the Current Multiplet.}

We began section 2.1 by postulating non-local conserved currents $J_{a}^{\mu}(x)$ for the charges $Q_{a} \in \mathcal{A}$. It is natural to suppose that the complete set of these non-local currents comprise a representation of the double algebra $\mathcal{D}(\mathcal{A})$, which we will refer to as the current representation $\rho_{J}$.

For the elements of $\mathcal{A}$, the current representation is defined as arising through the adjoint action:

$$
a d_{e_{a}}\left(Q_{b}\right)=\tilde{f}_{a b}^{c} Q_{c} \equiv \rho_{J}\left(e_{a}\right)_{b}^{c} Q_{c}
$$

The local form (2.67) can be taken as:

$$
\operatorname{ad}_{e_{a}}\left(J_{b}^{\mu}(x)\right)=\widetilde{f}_{a b}^{c} J_{c}^{\mu}(x)
$$

The current representation of elements of the dual algebra $\mathcal{A}^{*}$ on the non-local currents may be computed directly from the definition (2.52). One has:

$$
\begin{gathered}
R_{-\pi}\left(J_{a}^{\mu}(x)\right)=e^{n}\left(J_{a}^{\mu}(x)\right) s^{\prime}\left(e_{n}\right) \\
R_{\pi}\left(J_{a}^{\mu}(x)\right)=s\left(e_{n}\right) e^{n}\left(J_{a}^{\mu}(x)\right)
\end{gathered}
$$


The field $J_{a}^{\prime \mu}(x)$ is related to $J_{a}^{\mu}(x)$ by a rotation: $J_{a}^{\prime \mu}(x)=R_{-\pi}\left(J_{a}^{\mu}(x)\right)$. Thus the string for $J_{a}^{\prime \mu}(x)$ originates at $+\infty$ and ends at $x$. Deforming the string such that it first goes to $-\infty$ before continuing to $x$, one finds:

$$
J_{a}^{\prime \mu}(x)=J_{b}^{\mu}(x) s^{\prime}\left(\Theta_{a}^{b}\right)
$$

Comparing (2.70) with (2.69) and using (2.23), one finds:

$$
e^{n}\left(J_{a}^{\mu}(x)\right)=\mu_{a}^{n b} J_{b}^{\mu}(x) \equiv \rho_{J}\left(e^{n}\right)_{a}^{b} J_{b}^{\mu}(x) .
$$

The current representation defined in eqs. (2.68) and (2.71) indeed satisfies the complete set of relations of $\mathcal{D}(\mathcal{A})$. The proof is given in appendix $\mathrm{B}$.

An important aspect of the development in section 2.1 was the interpretation of $\Theta_{a}^{b}$ as a braiding operator. The fact that the currents are in the current representation, along with the general braiding relations (2.46), leads directly to a verification of the above interpretation of $\Theta_{a}^{b}$. Indeed we have:

$$
\begin{aligned}
J_{a}^{\mu}(x) \Phi_{\Lambda}(y) & =\Phi_{e_{d} \cdot \Lambda}(y) e^{d}\left(J_{a}^{\mu}(x)\right) \quad ; x>y \\
& =\mu_{a}^{d b} \Phi_{e_{d} \cdot \Lambda}(y) J_{b}^{\mu}(x) \\
& =a d_{\Theta_{a}^{b}}\left(\Phi_{\Lambda}(y)\right) J_{b}^{\mu}(x) .
\end{aligned}
$$

This illustrates once more the relation between braiding and the comultiplication.

Finally, we use the formulas (2.69) to derive a spin-statistics relation. The spin is defined through a rotation of $2 \pi$ : $\mathcal{S} \operatorname{pin}\left(J_{a}^{\mu}\right) \equiv R_{\pi}^{2}\left(J_{a}^{\mu}\right)$. Using eq. (2.69) one deduces,

$$
R_{\pi}^{2}\left(J_{a}^{\mu}\right)=a d_{s\left(\Theta_{a}^{d}\right)}\left(J_{d}^{\mu}\right)
$$

More explicitly, let us introduce the inverse braiding matrix $\bar{R}_{a b}^{c d}=\mu_{a}^{i b} s_{i}^{j} \widetilde{f}_{j c}^{d}$ characterized by $\bar{R}_{k c}^{b m} R_{a m}^{k d}=\delta_{c}^{d} \delta_{a}^{b}$. Then,

$$
\mathcal{S} \operatorname{pin}\left(J_{a}^{\mu}\right) \equiv \mathcal{S}_{a}^{b} J_{b}^{\mu}=\bar{R}_{a d}^{d b} J_{b}^{\mu} .
$$

Equivalently, the spin matrix is characterized by:

$$
\mathcal{S}_{n}^{m} R_{a m}^{n b}=\delta_{a}^{b}
$$

Eq. (2.75) is represented in figure 8. 


\section{Fig.8}

For currents with definite Lorentz spin, $\mathcal{S}_{a}^{b}$ is diagonal:

$$
R_{\pi}^{2}\left(J_{a}(x)\right)=e^{2 \pi i s_{a}} J_{a}(x)
$$

where $s_{a}$ is the Lorentz spin of the field $J_{a}(x) \bmod 1$.

\subsection{Twisting and Klein factors.}

It is well known that statistics of fields can be modified by so-called Klein factors. If the original field operators $\Phi_{j}\left(x_{j}\right)$ satisfy the braiding relation:

$$
\Phi_{1}\left(x_{1}\right) \Phi_{2}\left(x_{2}\right)=\mathcal{R}_{12} \Phi_{2}\left(x_{2}\right) \Phi_{1}\left(x_{1}\right) \quad ; \quad x_{1}<x_{2}
$$

then the fields $\widetilde{\Phi}_{j}\left(x_{j}\right)$ modified by the Klein factors satisfy the following braiding relation:

$$
\widetilde{\Phi}_{1}\left(x_{1}\right) \widetilde{\Phi}_{2}\left(x_{2}\right)=\widetilde{\mathcal{R}}_{12} \widetilde{\Phi}_{2}\left(x_{2}\right) \widetilde{\Phi}_{1}\left(x_{1}\right) \quad ; \quad x_{1}<x_{2}
$$

with

$$
\widetilde{\mathcal{R}}_{12}=F_{12} \mathcal{R}_{12} F_{21}^{-1}
$$

for some $F$. This modification corresponds to a gauge transformation of the braiding matrix $R_{12}$. The new braiding matrix is still a solution of the Yang-Baxter equation provided that the factor $F$ satisfies a 2-cocycle relation:

$$
(1 \otimes \Delta)(F)(1 \otimes F)=(\Delta \otimes 1)(F)(F \otimes 1)
$$

Since, as we explained in the previous sections, the comultiplication of the quantum symmetry algebra is encoded in the braiding relations, twisting the braidings will imply a twisting of the comultiplication. The new comultiplication $\widetilde{\Delta}$ is given by:

$$
\widetilde{\Delta}(a)=F^{-1} \Delta(a) F \quad ; \quad a \in \mathcal{A}
$$

It is still coassociative thanks to the cocycle condition (2.80). This twisting is a particular example of the Drinfel'd twist for quasi-Hopf algebras [25].

The simplest construction of Klein factors is provided by the cocycle operators involved in the vertex operator representation of current algebras. This amounts to multiplying the fields by operators with appropriate commutation relations which are encoded into a 2cocycle. 


\subsection{Remarks}

We conclude this section by emphasizing that, as we have developed them, the algebras $\mathcal{A}$ and $\mathcal{A}^{*}$ are on completely different footing. The elements of $\mathcal{A}$ are integrals of conserved currents, and the action of $e_{a}$ on a field is a generic adjoint action. This is not true for elements of $\mathcal{A}^{*}$. This fact is quite clear in the current representation, when one considers the integrated form of (2.71). This is not an adjoint action since

$$
e^{i}\left(Q_{a}\right)=\mu_{a}^{i b}\left(Q_{b}\right) \neq a d_{e^{i}}\left(Q_{a}\right) \equiv m_{c d}^{i} e^{c} Q_{a} s\left(e^{d}\right)
$$

This asymmetry between the algebras $\mathcal{A}$ and $\mathcal{A}^{*}$ is quite similar in spirit to what occurs for the finite quantum group symmetry of minimal conformal field theory [16] [17]. There, one Borel subalgebra $\mathcal{B}_{+}$of $S L(2)_{q}$ is realized as screening operators, and are thus integrals of conserved currents. The other half of $S L(2)_{q}$ arises as 'contour annihilation' operators, which are defined operationally via the conformal transformations of the field multiplets. Thus in the conformal field theory context $\mathcal{A}=\mathcal{B}_{+}$and the full $S L(2)_{q}$ algebra arises in the double $\mathcal{D}(\mathcal{A})$. In this paper the action of $e^{a} \in \mathcal{A}^{*}$ is defined operationally via the finite rotations $R_{ \pm \pi}$ rather than conformal transformations, as the latter are not generally

symmetries of the models. We remark however that for the affine $\widehat{S L(2)})_{q}$ symmetry of the sine-Gordon theory, the complete $\widehat{S L(2)})_{q}$ algebra is realized in terms of conserved currents and thus constitutes that algebra $\mathcal{A}$, which is quite different than the situation in conformal field theory; in this model one must actually deal with $\left.\mathcal{D}(\widehat{S L(2)})_{q}\right)$.

\subsection{Yangian Symmetry}

In this subsection we illustrate the above construction for the case of Yangian symmetry. As described in the introduction, integrable quantum field theories which are invariant under some finite Lie group $G$ generically have a larger symmetry corresponding to the Yangian $\mathcal{Y}$.

Let $J_{\mu}^{a}(x), a=1, . ., \operatorname{dim}(G)$ denote the local currents for the symmetry $G$, and $Q_{0}^{a}$ the conserved charges which generate the Lie algebra of $G$ 2:

$$
\left[Q_{0}^{a}, Q_{0}^{b}\right]=f^{a b c} Q_{0}^{c}
$$

${ }^{2}$ For $G=S L(2)$ we take $f^{a b c}=i \epsilon^{a b c}$. 
The non-local currents which generate the additional Yangian conserved charges are given by [6] [7]

$$
\mathcal{J}_{\mu}^{a}(x)=z(\delta) \epsilon_{\mu \nu} J_{\nu}^{a}(x)-\alpha f^{a b c} J_{\mu}^{b}(x) \int_{-\infty}^{x-\delta} J_{t}^{c}(y) d y
$$

In this expression, $z(\delta)$ is a constant which serves to remove the singularity at $\delta=0$. The current $\mathcal{J}_{\mu}^{a}$ is normalized such that the constant $\alpha$ is

$$
\alpha=-\frac{2 \pi i}{c_{A}}
$$

where $c_{A}$ is the Casimir in the adjoint representation: $f^{a b c} f^{b c d}=-c_{A} \delta^{a d} . \quad\left(c_{A}=2\right.$ for $\mathrm{SL}(2)$.) Let $Q_{1}^{a}$ denote the conserved charge for the non-local current, i.e. $Q_{1}^{a}=$ $\int_{-\infty}^{\infty} d x \mathcal{J}_{t}^{a}(x)$. The charge $Q_{1}^{a}$ is $G$-covariant:

$$
\left[Q_{0}^{a}, Q_{1}^{b}\right]=f^{a b c} Q_{1}^{c}
$$

One may easily apply the general construction in sections 2.1-2.3. The result is

$$
\begin{aligned}
& \Delta\left(Q_{0}^{a}\right)=Q_{0}^{a} \otimes 1+1 \otimes Q_{0}^{a} \\
& \Delta\left(Q_{1}^{a}\right)=Q_{1}^{a} \otimes 1+1 \otimes Q_{1}^{a}+\alpha f^{a b c} Q_{0}^{b} \otimes Q_{0}^{c} \\
& \epsilon\left(Q_{0}^{a}\right)=\epsilon\left(Q_{1}^{a}\right)=0 \\
& s\left(Q_{0}^{a}\right)=-Q_{0}^{a} ; \quad s\left(Q_{1}^{a}\right)=-Q_{1}^{a}+\alpha f^{a b c} Q_{0}^{b} Q_{0}^{c} .
\end{aligned}
$$

The adjoint actions are

$$
\begin{aligned}
& a d_{Q_{0}^{a}}(\Phi)=\left[Q_{0}^{a}, \Phi\right] \\
& a d_{Q_{1}^{a}}(\Phi)=\left[Q_{1}^{a}, \Phi\right]-\alpha f^{a b c}\left[Q_{0}^{b}, \Phi\right] Q_{0}^{c} .
\end{aligned}
$$

The equations (2.83), (2.86), and (2.87) are the defining relations of the Yangian $\mathcal{Y}$, which plays the role of the Hopf algebra $\mathcal{A}$ above. The Yangian, as defined by Drinfel'd [21], is a deformation of the positive half of the affine Lie algebra, with deformation parameter $\alpha(\alpha=0$ is the undeformed case). Though the relations (2.83), (2.86) are undeformed, the deformation (2.87) of the comultiplication implies that other relations of the affine Lie algebra must be deformed. Consider for example the case of $\operatorname{SL}(2)$. Define $Q_{2}^{a}=$ $-f^{a b c} Q_{1}^{b} Q_{1}^{c}$. (For $\mathrm{SL}(2)$ this definition is equivalent to $\left[Q_{1}^{a}, Q_{1}^{b}\right]=f^{a b c} Q_{2}^{c}$.) Then one has deformed Serre relations 21$]^{3}$

$$
\left[Q_{2}^{a}, Q_{1}^{b}\right]+\left[Q_{2}^{b}, Q_{1}^{a}\right]=\alpha^{2}\left(f^{a c d}\left\{Q_{0}^{b}, Q_{0}^{c}, Q_{1}^{d}\right\}+f^{b c d}\left\{Q_{0}^{a}, Q_{0}^{c}, Q_{1}^{d}\right\}\right),
$$

3 The following specific form of the deformed Serre relations is only valid for the SL(2) Yangian. 
where \{\} denotes the symmetrized product:

$$
\left\{x_{1}, x_{2}, \ldots, x_{n}\right\}=\frac{1}{n !} \sum_{i_{1} \neq i_{2} \neq \cdots \neq i_{n}} x_{i_{1}} x_{i_{2}} \cdots x_{i_{n}} .
$$

Obviously, for the usual affine Lie algebra the left hand side of (2.89) is zero.

We define $\mathcal{Y}^{*}$ as the dual algebra to $\mathcal{Y}$, and $\mathcal{D}(\mathcal{Y})$ as the Yangian double. $\mathcal{D}(\mathcal{Y})$ is generated by elements $Q_{-1}^{a}$. The algebraic relations in $\mathcal{D}(\mathcal{Y})$ can be determined from (2.49), (2.50) with $\left(Q_{0}^{a}\right)^{*} \equiv 2 \alpha Q_{-1}^{a}$, as was done in [10]. One thereby understands $\mathcal{D}(\mathcal{Y})$ as a deformation of the full affine Lie algebra. We will give a different presentation of $\mathcal{D}(\mathcal{Y})$ in the subsequent sections. For now, we illustrate the geometrical meaning of the quantum double for the current multiplet. From (2.71) and (2.87) one obtains

$$
Q_{-1}^{a}\left(\mathcal{J}_{\mu}^{b}(x)\right)=-\frac{1}{2} f^{a b c} J_{\mu}^{c}(x)
$$

Thus one sees that $\left(Q_{0}^{a}\right)^{*}$ acting on $\mathcal{J}_{\mu}^{b}(x)$ essentially removes the string, leaving $-\alpha f^{a b c} J_{\mu}^{c}(x)$. 


\section{Dynamical Properties}

In this section we describe how the affine quantum group symmetry fixes the S-matrix. We also discuss some aspects of the form factors. As stated in the introduction, the basic example we develop is the SL(2) Yangian.

\subsection{The S-matrix from the Universal $\mathcal{R}$-matrix}

The spectrum of the basic SL(2) Yangian invariant model consists of a doublet of solitons, transforming in the 2-dimensional representation of SL(2). Formulated as a currentcurrent perturbation of the WZW model (1.1), these solitons are created by the only chiral (or antichiral) primary fields of the WZW theory, which are in the 2-dimensional representation and have Lorentz spin $\pm 1 / 4[18]$. Denote the single particle states as $|\theta\rangle \in V$, where $V \simeq \mathbb{C}^{2}$ is the 2 -dimensional vector space, and $\theta$ is the rapidity:

$$
E=m \cosh (\theta) ; \quad P=m \sinh (\theta) .
$$

The representation of the charges $Q_{0}^{a}$ on the states is taken to be

$$
\rho_{V}\left(Q_{0}^{a}\right)|\theta\rangle=s^{a}|\theta\rangle
$$

where $s^{a}=\sigma^{a} / 2 .\left(\sigma^{a}\right.$ are the Pauli spin matrices. $)$

The representation of the charges $Q_{1}^{a}$ on the states can be deduced as follows. It was shown in [10] that one has the following fundamental relation between the energy momentum tensor $T_{\mu \nu}(x)$ and the local currents $J_{\mu}^{a}(x)$ :

$$
\left[Q_{1}^{a}, T_{\mu \nu}(x)\right]=-\frac{1}{2}\left(\epsilon_{\mu \alpha} \partial_{\alpha} J_{\nu}^{a}(x)+\epsilon_{\nu \alpha} \partial_{\alpha} J_{\mu}^{a}(x)\right)
$$

The above relation was proven by comparing the form factors of $T_{\mu \nu}$ and $J_{\mu}^{a}$, and using the Ward identities for the affine quantum group symmetry. The relation (3.3) can also be established by showing that $\left[Q_{1}^{a}, T_{\mu \nu}(x)\right]$ is local with respect to $J_{\mu}^{a}$; Lorentz and $\operatorname{SL}(2)$ covariance then fixes the right hand side of $(3.3)$. Let $L$ be the generator of Lorentz boost:

$$
L=-\int d x x T_{t t}(x) \quad(t=0)
$$

Then integration of $(3.3)$ yields

$$
\left[L, Q_{1}^{a}\right]=-Q_{0}^{a} .
$$


On shell, a finite Lorentz boost of rapidity $\beta$ shifts $\theta \rightarrow \theta-\beta$. Therefore, on shell, $L=\mathcal{L} \equiv-\frac{\partial}{\partial \theta}$. One thus deduces that

$$
\rho_{V}^{\theta}\left(Q_{1}^{a}\right)|\theta\rangle=\theta s^{a}|\theta\rangle
$$

More generally, the role of the Lorentz boost operator in the theory of affine quantum groups can be formulated algebraically as follows. Define $t_{\theta}$ to be an automorphism of $\mathcal{D}(\mathcal{A})$ such that $\rho_{V}^{\theta}(a)=\rho_{V}\left(t_{\theta}(a)\right), a \in \mathcal{D}(\mathcal{A})$. In the case of the Yangian this automorphism takes the form 21]

$$
t_{\theta}\left(Q_{1}^{a}\right)=Q_{1}^{a}+\theta Q_{0}^{a}, \quad t_{\theta}\left(Q_{0}^{a}\right)=Q_{0}^{a} .
$$

The automorphism $t_{\theta}$ corresponds to a finite Lorentz boost of rapidity $\theta: t_{\theta}\left(P_{ \pm}\right)=e^{\mp \theta} P_{ \pm}$, where $P_{ \pm}$are the light-cone components of the momentum. A Lorentz boost of rapidity $\theta$ is equivalent to a Euclidean rotation by the same angle. In the algebra $\mathcal{A}$, one has

$$
[L, a]=\mathcal{L}\left(t_{\theta}(a)\right)
$$

The fact that the non-local charges $Q_{1}^{a}$ act on the states in this momentum dependent fashion is the primary reason why they provide strong dynamical constraints on the theory. We now construct the S-matrix using only the quantum symmetry by relating it to the universal $\mathcal{R}$-matrix. The two particle to two particle $\mathrm{S}$-matrix is an operator

$$
S_{12}\left(\theta_{1}-\theta_{2}\right): \quad V_{1} \otimes V_{2} \rightarrow V_{2} \otimes V_{1}
$$

It is required to commute with the quantum symmetry:

$$
S_{12}\left(\theta_{1}-\theta_{2}\right) \rho_{V_{1}}^{\theta_{1}} \otimes \rho_{V_{2}}^{\theta_{2}}\left(\Delta^{\prime}(a)\right)=\rho_{V_{1}}^{\theta_{1}} \otimes \rho_{V_{2}}^{\theta_{2}}(\Delta(a)) S_{12}\left(\theta_{1}-\theta_{2}\right), \quad a \in \mathcal{A} .
$$

The above symmetry equation is enough to fix the S-matrix up to an overall scalar function of $\theta_{12} \equiv \theta_{1}-\theta_{2}$, and the solution automatically satisfies the Yang-Baxter equation. This follows from well-known results in the theory of affine quantum groups[21] [23]. In the case of the Yangian it fixes $S \propto(\theta+\alpha P)$, where $P$ is the permutation operator $\left(P v_{1} \otimes v_{2}=\right.$ $\left.v_{2} \otimes v_{1}\right)$. Since the dual algebra $\mathcal{A}^{*}$ has so far played no role in the equation (3.9), it is natural to suppose that the full quantum double structure fixes in addition the overall scalar factor, as was argued by Smirnov[12]. 
Given the representation $\rho_{V}^{\theta}$ of $\mathcal{A}$, and the relations (2.49), one can in principle deduce the representation $\rho_{V}^{\theta}$ in the full quantum double $\mathcal{D}(\mathcal{A})$. Consider then evaluating the universal $\mathcal{R}$-matrix in this representation:

$$
R^{V_{1} V_{2}}\left(\theta_{1}, \theta_{2}\right)=\sum_{a} \rho_{V_{1}}^{\theta_{1}}\left(e_{a}\right) \otimes \rho_{V_{2}}^{\theta_{2}}\left(e^{a}\right)
$$

The matrix $R^{V_{1} V_{2}}$ satisfies several important identities as a consequence of the quantum double structure. Where there is no chance for confusion, we will simplify the notation and write $R_{12}=R^{V_{1} V_{2}}$. Due to the automorphism $t_{\theta}, R_{12}$ is only a function of $\theta_{12}$. By construction it satisfies the $\mathcal{A}$-symmetry equations (3.9). It also automatically satisfies the rapidity-dependent Yang-Baxter equation, as a consequence of (A.9):

$$
R_{12}\left(\theta_{12}\right) R_{13}\left(\theta_{13}\right) R_{23}\left(\theta_{23}\right)=R_{23}\left(\theta_{23}\right) R_{13}\left(\theta_{13}\right) R_{12}\left(\theta_{12}\right)
$$

There are two additional relations satisfied by $R_{12}(\theta)$ which are related to crossing symmetry and unitarity. The unitarity condition reads

$$
R_{12}(\theta) R_{21}(-\theta)=1
$$

(In the above equation $R_{21}=P R_{12} P$.) A proof of 3.12 may be given along the following lines. Since $R$ satisfies the equation (A.6), then $R^{\prime} \equiv P R P$ satisfies $R^{\prime} \Delta=\Delta^{\prime} R$. Since the equation (A.6) determines $R$ up to an overall scalar factor and assuming that the tensor product $V_{1} \otimes V_{2}$ is irreducible for generic values of $\theta, R^{\prime}$ must be proportional to the inverse of $R$ : $R_{12}(\theta) R_{21}(-\theta)=f(\theta)$, where $f(\theta)$ is some scalar function. Now using the quasi-triangularity property (A.11), one finds $(\Delta \otimes 1) R_{12} R_{21}=R_{23} R_{13} R_{31} R_{32}$. The latter implies $f(\theta)=(f(\theta))^{2}$, or $f(\theta)=1$.

The crossing symmetry property may be derived as follows. Suppose that in a particular representation of $\mathcal{A}$ the following formula is valid

$$
\rho_{V}^{\theta}\left(s^{\prime}(a)\right)=C\left(\rho_{V}^{\theta+i \pi}(a)\right)^{t} C^{-1}
$$

where $C$ is a constant 'charge conjugation' matrix, and $t$ denotes transpose. Indeed it is clear from (2.12), and from the equivalence of Lorentz boosts and Euclidean rotations, that the antipode $s^{\prime}$ should shift $\theta$ by $i \pi$. The transpose $t$ ensures $s^{\prime}(a b)=s^{\prime}(b) s^{\prime}(a)$. Then (A.14) implies

$$
R_{12}^{-1}(\theta)=C_{1} R_{12}^{t_{1}}(\theta+i \pi) C_{1}^{-1}
$$


thus

$$
\left(R_{12}(\theta)\right)\left(C_{1} R_{12}^{t_{1}}(\theta+i \pi) C_{1}^{-1}\right)=1 .
$$

Combining (3.12) and (3.15) on obtains the crossing relation

$$
R_{21}(\theta)=C_{1} R_{12}^{t_{1}}(i \pi-\theta) C_{1}^{-1}
$$

Let us now specialize to the Yangian. One finds $\rho_{V}^{\theta}\left(s^{\prime}(a)\right)=C\left(\rho_{V}^{\theta-\alpha}(a)\right)^{t} C^{-1}$, with

$$
C=\left(\begin{array}{cc}
0 & 1 \\
-1 & 0
\end{array}\right)
$$

(Recall $\alpha=-i \pi$.$) One may express R_{12}$ as

$$
R_{12}(\theta)=v(\theta)(\theta+\alpha P)
$$

where $v(\theta)$ is a scalar function[21]. Then (3.12) and (3.15) imply the following functional relations for $v(\theta)$ :

$$
\begin{aligned}
v(\theta) v(-\theta) & =\frac{1}{\alpha^{2}-\theta^{2}} \\
v(\theta) v(\theta-\alpha) & =\frac{1}{\theta^{2}-\alpha^{2}} \\
\Rightarrow \quad v(\theta) & =-v(-\theta-\alpha) .
\end{aligned}
$$

Related arguments for fixing overall scalar factors were given in [21] 13] 12].

The formula (3.18b) is equivalent to requiring that the quantum determinant is 1 . To see this, let $\widetilde{T}(\theta) \equiv \rho_{V}^{\theta}\left(e_{a}\right) \otimes e^{a}$ denote a matrix with elements $\widetilde{T}_{i j} \in \mathcal{A}^{*}, i, j \in\{1,2\}$. Then the quantum determinant of $\widetilde{T}$ is defined as 26]

$$
\begin{aligned}
\operatorname{Det}_{q}(\widetilde{T}(\theta)) \equiv \frac{1}{2}\left(\widetilde{T}_{11}(\theta) \widetilde{T}_{22}(\theta+\alpha)+\widetilde{T}_{22}(\theta) \widetilde{T}_{11}(\theta+\alpha)\right. \\
\left.-\widetilde{T}_{12}(\theta) \widetilde{T}_{21}(\theta+\alpha)-\widetilde{T}_{21}(\theta) \widetilde{T}_{12}(\theta+\alpha)\right) .
\end{aligned}
$$

Using the fact that $\rho_{V}(\widetilde{T})=R$, one can show that $\rho_{V}\left(\operatorname{Det}_{q}(\widetilde{T})\right)=1$ implies $(3.18 \not)$.

In order to compare with the conventional physical S-matrix, one must consider a twisted $R$-matrix along the lines developed in section 2.6. This is due to the fact that the soliton fields which create the particles have Lorentz spin $\pm 1 / 4$, and therefore have non-trivial braiding among themselves and with the local currents $J_{a}^{\mu}(x)$. Let $\left\{Q_{0}^{3}, Q_{0}^{ \pm}\right\}$ denote a basis for the charges $Q_{0}^{a}$, with $\left[Q_{0}^{3}, Q_{0}^{ \pm}\right]= \pm 2 Q_{0}^{ \pm},\left[Q_{0}^{+}, Q_{0}^{-}\right]=Q_{0}^{3}$, and similarly 
for $Q_{1}^{a}$. Let $J_{\mu}^{ \pm}(x), J_{\mu}^{3}(x)$ denote the corresponding currents, and $\Psi_{V}(x)$ the soliton fields. It is clear from a bosonized description (see e.g. [4] and references therein) that one has

$$
\begin{aligned}
& J_{\mu}^{ \pm}(x) \Psi_{V}(y)=(-1)^{ \pm \widehat{Q}_{0}^{3}}\left(\Psi_{V}(y)\right) J_{\mu}^{ \pm}(x) \quad x>y \\
& J_{\mu}^{3}(x) \Psi_{V}(y)=\Psi_{V}(y) J_{\mu}^{3}(x) .
\end{aligned}
$$

In accordance with the general principles outlined in section 2, in determining the S-matrix one should take the twisted comultiplication $\widetilde{\Delta}$ :

$$
\begin{aligned}
& \widetilde{\Delta}\left(Q_{0}^{3}\right)=Q_{0}^{3} \otimes 1+1 \otimes Q_{0}^{3} \\
& \widetilde{\Delta}\left(Q_{0}^{ \pm}\right)=Q_{0}^{ \pm} \otimes 1+(-1)^{ \pm Q_{0}^{3}} \otimes Q_{0}^{ \pm} \\
& \widetilde{\Delta}\left(Q_{1}^{ \pm}\right)=Q_{1}^{ \pm} \otimes 1+(-1)^{ \pm Q_{0}^{3}} \otimes Q_{1}^{ \pm} \mp \frac{1}{2} \alpha\left((-1)^{ \pm Q_{0}^{3}} Q_{0}^{3} \otimes Q_{0}^{ \pm}-Q_{0}^{ \pm} \otimes Q_{0}^{3}\right) \\
& \widetilde{\Delta}\left(Q_{1}^{3}\right)=Q_{1}^{3} \otimes 1+1 \otimes Q_{1}^{3}+\alpha\left((-1)^{Q_{0}^{3}} Q_{0}^{-} \otimes Q_{0}^{+}-(-1)^{-Q_{0}^{3}} Q_{0}^{+} \otimes Q_{0}^{-}\right)
\end{aligned}
$$

One may check that this twisted comultiplication is still a homomorphism of the Yangian relations (2.83) and (2.86), i.e. (A.1d) holds with $\Delta \rightarrow \widetilde{\Delta}$. This twisted comultiplication may also be understood as a consequence of the fact that the Yangian invariant model arises as the $q \rightarrow-1$ limit of the $\widehat{S L(2)}$ invariant sine-Gordon model [4].

The above twist of the comultiplication implies (via (2.21) with $\Delta \rightarrow \widetilde{\Delta}$ ) that the antipodes are modified to

$$
\begin{aligned}
& s\left(Q_{0}^{3}\right)=-Q_{0}^{3}, \quad s\left(Q_{0}^{ \pm}\right)=-(-1)^{\mp Q_{0}^{3}} Q_{0}^{ \pm} \\
& s\left(Q_{1}^{3}\right)=-Q_{1}^{3}-\alpha Q_{0}^{3}, \quad s\left(Q_{1}^{ \pm}\right)=(-1)^{\mp Q_{0}^{3}}\left(-Q_{1}^{ \pm}-\alpha Q_{0}^{ \pm}\right) .
\end{aligned}
$$

Consequently, one finds that $(\underline{3.13})$ is valid, but with $C \rightarrow \widetilde{C}$,

$$
\widetilde{C}=\left(\begin{array}{ll}
0 & 1 \\
1 & 0
\end{array}\right)
$$

Repeating the above analysis with the twisted $R$-matrix $\widetilde{R}$, one finds it is still crossing symmetric and unitary. $\widetilde{R}_{12}$ may be expressed as

$$
\widetilde{R}_{12}=\widetilde{v}(\theta)\left(\begin{array}{cccc}
-\alpha-\theta & 0 & 0 & 0 \\
0 & \theta & -\alpha & 0 \\
0 & -\alpha & \theta & 0 \\
0 & 0 & 0 & -\alpha-\theta
\end{array}\right)
$$

The functional relations for $\widetilde{v}$ are now changed to

$$
\widetilde{v}(\theta) \widetilde{v}(-\theta)=1, \quad \widetilde{v}(\theta)=\widetilde{v}(-\alpha-\theta) .
$$


The functional relations (3.25), together with some knowledge of the asymptotic development of $\widetilde{R}(\theta)$ as $\theta \rightarrow \pm \infty$, are enough to determine a solution. Expressing $\widetilde{R}_{12}=\rho_{V}\left(e_{a}\right) \otimes \rho_{V}^{-\theta}\left(e^{a}\right)$, and noting that $\rho_{V}^{-\theta}\left(e^{a}\right)$ only has zero or negative powers of $\theta$, one sees that $\widetilde{v}(\theta)=\mathbb{C}\left[\left[\theta^{-1}\right]\right] / \theta$. Thus

$$
\widetilde{v}(\theta \rightarrow \pm \infty) \rightarrow \pm \frac{i}{\theta}
$$

In the limit $\theta \rightarrow \pm \infty, \widetilde{R}(\theta)$ becomes the braiding matrix of the solitons:

$$
\widetilde{R}_{12}(\theta \rightarrow \pm \infty) \rightarrow \pm \operatorname{diag}(-i, i, i,-i) .
$$

A solution to the functional equations (3.25), which has the proper asymptotic development (3.26), may be constructed iteratively to yield an infinite product expression:

$$
\widetilde{v}(\theta)=\frac{1}{\theta-\alpha} \prod_{j=1}^{\infty}\left[\left(\frac{-\alpha(2 j)+\theta}{-\alpha(2 j)-\theta}\right)\left(\frac{-\alpha(2 j+1)-\theta}{-\alpha(2 j+1)+\theta}\right)\right] .
$$

Using standard identities this may be expressed as

$$
\widetilde{v}(\theta)=\frac{1}{\theta+\alpha} \frac{\Gamma(1 / 2-\theta / 2 \alpha) \Gamma(\theta / 2 \alpha)}{\Gamma(1 / 2+\theta / 2 \alpha) \Gamma(-\theta / 2 \alpha)} .
$$

One may check using Sterling's formula that $\widetilde{v}(\theta)$ has the asymptotics (3.26). Comparing with the known physical S-matrix, we conclude that this S-matrix is precisely characterized by the twisted universal $\mathcal{R}$-matrix:

$$
S_{12}(\theta)=\widetilde{R}_{12}(\theta) .
$$

\subsection{Multiplicative Presentations of the Quantum Double from Vertex Operators}

Let us formally introduce generalized creation-annihilation operators $Z(\theta)$, valued in the particle vector space $V$, such that the multiparticle asymptotic states correspond to the action of $Z(\theta)$ 's on the vacuum:

$$
\left|\theta_{1}, \ldots, \theta_{n}\right\rangle=Z\left(\theta_{1}\right) \cdots Z\left(\theta_{n}\right)|0\rangle .
$$

Though we have only formally introduced the operators $Z(\theta)$, they may be given the following interpretation. Consider a free scalar field theory. Normally this field may be expanded into creation-annihilation operators $a(\theta), a^{\dagger}(\theta)$. This may be written as

$$
\phi(x)=\oint_{\mathcal{C}} d \theta Z(\theta) e^{-i p(\theta) \cdot x},
$$


where the contour $\mathcal{C}$ in the $\theta$-plane runs along the real axis and returns along the line $\theta=i \pi$. Namely, $Z(\theta)=a(\theta)$ and $Z(\theta+i \pi)=a^{\dagger}(\theta)$ for $\theta$ real. The $Z(\theta)$ operators may roughly be thought of as a generalization of this to an interacting theory.

The operators $Z(\theta)$ are defined to be characterized by their intertwining relations:

$$
\begin{aligned}
a d_{e_{a}}(Z(\theta)) & =\rho_{V}^{\theta}\left(e_{a}\right) Z(\theta) \\
e^{a}(Z(\theta)) & =\rho_{V}^{\theta}\left(e^{a}\right) Z(\theta) .
\end{aligned}
$$

They are thus by definition vertex operators for the affine quantum group. Such vertex operators were defined and studied in [13]. They are also defined to have S-matrix exchange relations:

$$
Z_{1}\left(\theta_{1}\right) Z_{2}\left(\theta_{2}\right)=S_{12}\left(\theta_{12}\right) Z_{2}\left(\theta_{2}\right) Z_{1}\left(\theta_{1}\right)
$$

where the subscripts refer to the vector spaces $V_{1}, V_{2}$. For simplicity we here ignore complications due to Klein factors and take $S_{12}(\theta)=R_{12}(\theta)$. By applying $a d_{e_{a}}$ to both sides of (3.34) one derives that $S_{12}$ must satisfy the defining relations of the universal $\mathcal{R}$-matrix, so that (3.34) may be viewed as a consequence of (3.33).

The vacuum is defined to satisfy

$$
\begin{array}{ll}
e_{a}|0\rangle=\epsilon_{a}|0\rangle, & e^{a}|0\rangle=\epsilon^{a}|0\rangle \\
\langle 0| e_{a}=\langle 0| \epsilon_{a}, & \langle 0| e^{a}=\langle 0| \epsilon^{a},
\end{array}
$$

which is the statement that the vacuum is 'annihilated' by the elements of $\mathcal{D}(\mathcal{A})$. It is evident from the properties in the appendix that the counits form one-dimensional representations of $\mathcal{D}(\mathcal{A}): \epsilon_{a} \epsilon_{b}=m_{a b}^{c} \epsilon_{c}, \epsilon^{a} \epsilon^{b}=\mu_{c}^{b a} \epsilon^{c}$. One also has

$$
\begin{aligned}
e_{a} Z\left(\theta_{1}\right) \cdots Z\left(\theta_{n}\right) & =\mu_{a}^{b c} a d_{e_{b}}\left(Z\left(\theta_{1}\right) \cdots Z\left(\theta_{n}\right)\right) e_{c} \\
e^{a} Z\left(\theta_{1}\right) \cdots Z\left(\theta_{n}\right) & =m_{b c}^{a} e^{b}\left(Z\left(\theta_{1}\right) \cdots Z\left(\theta_{n}\right)\right) e^{c} \\
Z\left(\theta_{1}\right) \cdots Z\left(\theta_{n}\right) s\left(e_{a}\right) & =\mu_{a}^{b c} s\left(e_{b}\right) a d_{e_{c}}\left(Z\left(\theta_{1}\right) \cdots Z\left(\theta_{n}\right)\right) \\
Z\left(\theta_{1}\right) \cdots Z\left(\theta_{n}\right) s\left(e^{a}\right) & =m_{b c}^{a} s\left(e^{b}\right) e^{c}\left(Z\left(\theta_{1}\right) \cdots Z\left(\theta_{n}\right)\right) .
\end{aligned}
$$

Combined with (3.35), and the analog of (2.33), the above relations imply for instance

$$
\begin{aligned}
e_{a} Z\left(\theta_{1}\right) \cdots Z\left(\theta_{n}\right)|0\rangle & =a d_{e_{a}}\left(Z\left(\theta_{1}\right) \cdots Z\left(\theta_{n}\right)\right)|0\rangle \\
& =\rho_{V_{1} \cdots V_{n}}\left(e_{a}\right) Z\left(\theta_{1}\right) \cdots Z\left(\theta_{n}\right)|0\rangle,
\end{aligned}
$$

where

$$
\rho_{V_{1} \cdot V_{n}}\left(e_{a}\right)=\mu_{a}^{b_{1} c_{1}} \mu_{c_{1}}^{b_{2} c_{2}} \cdots \mu_{c_{n-2}}^{b_{n-1} b_{n}} \rho_{V_{1}}\left(e_{b_{1}}\right) \otimes \cdots \otimes \rho_{V_{n}}\left(e_{b_{n}}\right) .
$$


It was understood by Smirnov that the elements of $\mathcal{D}(\mathcal{A})$ can be reconstructed from the algebra of vertex operators. Here, we clarify some aspects of this idea. Define a matrix $L(\theta)$ of operators, with matrix elements

$$
L_{a}^{b}(\theta)=2 \pi i \underset{\theta^{\prime}=\theta-i \pi}{\operatorname{res}} Z_{a}(\theta) Z_{c}\left(\theta^{\prime}\right) C^{c b}
$$

It is not difficult to prove using only the exchange relation (3.34) and crossing symmetry (3.16) that $L$ satisfies the following relation

$$
S_{21}\left(\theta_{21}\right) L_{1}\left(\theta_{1}\right) S_{12}\left(\theta_{12}\right) L_{2}\left(\theta_{2}\right)=L_{2}\left(\theta_{2}\right) S_{21}\left(\theta_{21}\right) L_{1}\left(\theta_{1}\right) S_{12}\left(\theta_{12}\right)
$$

where $L_{1}=L \otimes 1, L_{2}=1 \otimes L$.

Relations of the kind (3.40) are well-known in the theory of quantum groups and are referred to as multiplicative presentations [21] [22]. Define two matrices of operators $T^{ \pm}(\theta)$ as follows

$$
\begin{aligned}
& T^{+}(\theta)=\sum_{a} e_{a} \rho_{V}^{\theta}\left(e^{a}\right) \\
& T^{-}(\theta)=\sum_{a} \rho_{V}^{\theta}\left(s^{\prime}\left(e_{a}\right)\right) e^{a} .
\end{aligned}
$$

The matrix elements of $T^{+}$are in $\mathcal{A}$, whereas those of $T^{-}$are in $\mathcal{A}^{*}$. From the Yang-Baxter equation (A.9) evaluated in the appropriate representations, one obtains

$$
\begin{aligned}
& R_{12}\left(\theta_{12}\right) T_{2}^{ \pm}\left(\theta_{2}\right) T_{1}^{ \pm}\left(\theta_{1}\right)=T_{1}^{ \pm}\left(\theta_{1}\right) T_{2}^{ \pm}\left(\theta_{2}\right) R_{12}\left(\theta_{12}\right) \\
& R_{12}\left(\theta_{12}\right) T_{2}^{+}\left(\theta_{2}\right) T_{1}^{-}\left(\theta_{1}\right)=T_{1}^{-}\left(\theta_{1}\right) T_{2}^{+}\left(\theta_{2}\right) R_{12}\left(\theta_{12}\right)
\end{aligned}
$$

One can also show using $(\underline{3.41})$ and the basic properties of $\mathcal{D}(\mathcal{A})$ that

$$
T^{ \pm}(\theta) s^{\prime}\left(T^{ \pm}(\theta)\right)=s^{\prime}\left(T^{ \pm}(\theta)\right) T^{ \pm}(\theta)=1
$$

and

$$
\Delta^{\prime}\left(T^{ \pm}(\theta)\right)=T^{ \pm}(\theta) \otimes T^{ \pm}(\theta)
$$

The algebra defined by (3.42)-(3.44) is isomorphic to $\mathcal{D}(\mathcal{A})$, provided one also imposes that the quantum determinant of $T^{ \pm}$is 1 . Finally define

$$
T(\theta) \equiv T^{+}(\theta) s^{\prime}\left(T^{-}(\theta)\right)
$$

Then using the relations (3.42) one can show

$$
R_{21}\left(\theta_{21}\right) T_{1}\left(\theta_{1}\right) R_{12}\left(\theta_{12}\right) T_{2}\left(\theta_{2}\right)=T_{2}\left(\theta_{2}\right) R_{21}\left(\theta_{21}\right) T_{1}\left(\theta_{1}\right) R_{12}\left(\theta_{12}\right) \text {. }
$$


If $T$ satisfies (3.46), then it is not difficult to show using the unitary property of $R_{12}$, that $T-1$ does also. The consistent identification is

$$
L=T-1
$$

This conclusion is reached by studying the counits. By construction $\epsilon(T)=1$. On the other hand, from the previously given definition of the counit one finds

$$
\epsilon\left(L_{a}^{b}(\theta)\right)=2 \pi i \underset{\theta^{\prime}=\theta-i \pi}{\operatorname{res}}\left\langle 0\left|Z_{a}(\theta) Z_{c}\left(\theta^{\prime}\right) C^{c b}\right| 0\right\rangle=0 .
$$

Note that the vertex operator algebra only yields the relation (3.40), thus one must assume that $L+1$ can be factorized as in (3.45) in order to extract the $\mathcal{D}(\mathcal{A})$ relations $(3.42)$.

The intertwining property 3.33 of the operators $Z(\theta)$ can also be derived from the exchange relation (3.34) and the reconstruction formula (3.39) of the operators $L(\theta)$. A simple computation gives :

$$
L_{1}\left(\theta_{1}\right) S_{12}\left(\theta_{12}\right) Z_{2}\left(\theta_{2}\right)=S_{12}\left(\theta_{12}\right) Z_{2}\left(\theta_{2}\right) L_{1}\left(\theta_{1}\right)
$$

and similarly with $T=L+1$. Eq. (3.49) is the multiplicative form of the intertwining relations (3.33). Notice that the set of relations (3.34), (3.40) and (3.49) is very reminiscent of the defining relation of the quantum cotangent bundle [27]. Finally, one can also show from (3.41) and (3.37) that

$$
\begin{aligned}
& T_{0}^{+}\left(\theta_{0}\right) Z_{1}\left(\theta_{1}\right) \cdots Z_{n}\left(\theta_{n}\right)|0\rangle=S_{10}\left(\theta_{10}\right) S_{20}\left(\theta_{20}\right) \cdots S_{n 0}\left(\theta_{n 0}\right) Z_{1}\left(\theta_{1}\right) \cdots Z_{n}\left(\theta_{n}\right)|0\rangle \\
& T_{0}^{-}\left(\theta_{0}\right) Z_{1}\left(\theta_{1}\right) \cdots Z_{n}\left(\theta_{n}\right)|0\rangle=S_{10}\left(\theta_{10}\right) S_{20}\left(\theta_{20}\right) \cdots S_{n 0}\left(\theta_{n 0}\right) Z_{1}\left(\theta_{1}\right) \cdots Z_{n}\left(\theta_{n}\right)|0\rangle .
\end{aligned}
$$

\subsection{Multiplicative Presentations of the Yangian Double}

In [10] the Yangian Double was studied using directly the formulas in appendix A. In this section we develop more fully the multiplicative presentation of the last subsection. Though it is evident from (3.41) that one can extract the $\mathcal{D}(\mathcal{A})$ algebra from $(3.42)$, this exercise is useful in providing a concrete basis for the generators where the relations are finite, and will make it evident that $\mathcal{D}(\mathcal{Y})$ is a deformation of the full affine Lie algebra.

Expand $T^{ \pm}(\theta)$ as power series in $\theta$ as follows:

$$
\begin{aligned}
& T^{+}(\theta)=1-2 \alpha \sum_{n=0}^{\infty} t^{(n)} \theta^{-n-1} \\
& T^{-}(\theta)=1+2 \alpha \sum_{n=0}^{\infty} t^{(-n-1)} \theta^{n} .
\end{aligned}
$$


In the relations (3.42) one can cancel the factor $v(\theta)$ in (3.17) from both sides of the equation to obtain

$$
\begin{aligned}
& {\left[T_{i j}^{ \pm}\left(\theta_{2}\right), T_{k l}^{ \pm}\left(\theta_{1}\right)\right]=\frac{\alpha}{\theta_{1}-\theta_{2}}\left(T_{k j}^{ \pm}\left(\theta_{1}\right) T_{i l}^{ \pm}\left(\theta_{2}\right)-T_{k j}^{ \pm}\left(\theta_{2}\right) T_{i l}^{ \pm}\left(\theta_{1}\right)\right)} \\
& {\left[T_{i j}^{+}\left(\theta_{2}\right), T_{k l}^{-}\left(\theta_{1}\right)\right]=\frac{\alpha}{\theta_{1}-\theta_{2}}\left(T_{k j}^{-}\left(\theta_{1}\right) T_{i l}^{+}\left(\theta_{2}\right)-T_{k j}^{+}\left(\theta_{2}\right) T_{i l}^{-}\left(\theta_{1}\right)\right) .}
\end{aligned}
$$

Let $t_{i j}^{(n)}, i, j=1,2$ denote the matrix elements of $t^{(n)}$. Then the above formulas imply

$$
\begin{aligned}
& {\left[t_{i j}^{(n)}, t_{k l}^{(m)}\right]=\frac{1}{2}\left(\delta_{i l} t_{k j}^{(m+n)}-\delta_{k j} t_{i l}^{(m+n)}\right)+\alpha \sum_{p \leq m-1}\left(t_{k j}^{(m-p-1)} t_{i l}^{(n+p)}-t_{k j}^{(n+p)} t_{i l}^{(m-p-1)}\right)} \\
& {\left[t_{i j}^{(-n)}, t_{k l}^{(-m)}\right]=\frac{1}{2}\left(\delta_{i l} t_{k j}^{(-m-n)}-\delta_{k j} t_{i l}^{(-m-n)}\right)} \\
& +\alpha \sum_{p \leq n-1}\left(t_{k j}^{(-m-p-1)} t_{i l}^{(p-n)}-t_{k j}^{(p-n)} t_{i l}^{(-m-p-1)}\right) \\
& {\left[t_{i j}^{(n)}, t_{k l}^{(-m)}\right]=\frac{1}{2}\left(\delta_{i l} t_{k j}^{(n-m)}-\delta_{k j} t_{i l}^{(n-m)}\right)} \\
& +\alpha \sum_{p \leq m i n(m-1, n-1)}\left(t_{k j}^{(p-m)} t_{i l}^{(n-p-1)}-t_{k j}^{(n-p-1)} t_{i l}^{(p-m)}\right), \\
& \Delta^{\prime}\left(t_{i j}^{(n)}\right)=t_{i j}^{(n)} \otimes 1+1 \otimes t_{i j}^{(n)}-2 \alpha \sum_{p \leq n-1} t_{i k}^{(p)} \otimes t_{k j}^{(n-1-p)} \\
& \Delta^{\prime}\left(t_{i j}^{(-n)}\right)=t_{i j}^{(-n)} \otimes 1+1 \otimes t_{i j}^{(-n)}+2 \alpha \sum_{p \leq n-1} t_{i k}^{(-p-1)} \otimes t_{k j}^{(-n+p)} .
\end{aligned}
$$

(Above, $t_{i j}^{(n)}\left(t_{i j}^{(-n)}\right)$ has $n \geq 0(n \geq 1)$.) In deriving these relations we used the identity $1 /\left(\theta-\theta^{\prime}\right)=\left(1+\sum_{n=1}^{\infty}\left(\theta^{\prime} / \theta\right)^{n}\right) / \theta$.

It is clear that when $\alpha=0$, the algebra $\mathcal{D}(\mathcal{Y})$ defined in (3.53) is isomorphic to the level $0 \mathrm{SL}(2)$ affine Lie algebra. The isomorphism is $t^{(n)}=\sum_{a=1}^{3} Q_{n}^{a} s^{a}, n \in \mathbb{Z}$, with $\left[Q_{n}^{a}, Q_{m}^{b}\right]=f^{a b c} Q_{n+m}^{c}$. Note also that when $\alpha \neq 0, \mathcal{Y}$ and $\mathcal{Y}^{*}$ are both deformations of the respective halves of the affine Lie algebra.

The elements $Q_{0}^{a}$, defined as $t^{(0)}=\sum_{a} Q_{0}^{a} s^{a}$, generate an $\operatorname{SL}(2)$ subalgebra of $\mathcal{D}(\mathcal{Y})$. It is possible to extract generators which are covariant with respect to this $\mathrm{SL}(2)$ out of the generators $t^{(n)}$. For the algebra $\mathcal{Y}$ this is well-known[21]. Here we describe how to do this for the full double. Let us first review the result for $\mathcal{Y}$. Define

$$
\begin{aligned}
t^{(0)} & =\sum_{a=1}^{3} Q_{0}^{a} s^{a} \\
t^{(1)} & =\sum_{a=1}^{3} Q_{1}^{a} s^{a}+\frac{\alpha}{4} Q_{0}^{a} Q_{0}^{a} .
\end{aligned}
$$


The additional $\alpha$-dependent term in $t^{(1)}$ is required for the comultiplication to close. From (3.54) one derives the first equations in (2.87), and from (3.53) one obtains (2.83) (2.86). The deformation of the Serre relations can be proven from (3.53) as follows. Consider for example $\left[Q_{2}^{-}, Q_{1}^{-}\right]$. This can be expressed in terms of the basic generators as $\left[Q_{2}^{-}, Q_{1}^{-}\right]=$ $\left[Q_{1}^{-},\left[Q_{1}^{3}, Q_{1}^{-}\right]\right]$. Expressing this in terms of $t$ 's, one finds $Q_{1}^{-}=2 t_{12}^{(1)}, Q_{1}^{3}=t_{11}^{(1)}-t_{22}^{(1)}$. Using the relations (3.53) one can thereby evaluate the above commutator:

$$
\left[Q_{2}^{-}, Q_{1}^{-}\right]=-\frac{\alpha^{2}}{2}\left(2 Q_{0}^{-} Q_{1}^{3} Q_{0}^{-}-Q_{0}^{-} Q_{0}^{3} Q_{1}^{-}-Q_{1}^{-} Q_{0}^{3} Q_{0}^{-}\right) .
$$

The latter is equivalent to (2.89). The other relations can be proven similarly.

For the full double $\mathcal{D}(\mathcal{Y})$, the situation is considerably more complicated, due to the fact that the $t^{(-n)}$, for $n \geq 1$, are infinite series in the covariant generators. To see this, consider starting with the definition $t^{(-1)}=\sum_{a} Q_{-1}^{a} s^{a}$. One finds that $\Delta^{\prime}\left(t^{(-1)}\right)$ does not close on $\Delta^{\prime}\left(Q_{-1}^{a}\right)$ to any finite order in $\alpha$. One must successively correct $t^{(-1)}$, order by order in $\alpha$, in order for $\Delta^{\prime}\left(t^{(-1)}\right)$ to close. Once this is done, then one finds that the $t^{(-n)}$, $n \geq 2$ can also be expressed in terms of $Q_{-1}^{a}$. In other words, the algebra $\mathcal{Y}^{*}$ is generated solely by the elements $Q_{-1}^{a}$, and is a deformation of the universal enveloping algebra of the negative half of the affine Lie algebra. We develop this to lowest orders in $\alpha$. Define

$$
\begin{aligned}
& t^{(-1)}=\sum_{a} Q_{-1}^{a} s^{a}+\frac{\alpha}{4} Q_{-1}^{a} Q_{-1}^{a}+\ldots \\
& t^{(-2)}=\sum_{a} Q_{-2}^{a} s^{a}+\frac{\alpha}{4}\left(Q_{-1}^{a} Q_{-2}^{a}+Q_{-2}^{a} Q_{-1}^{a}\right)+\ldots
\end{aligned}
$$

Then $\Delta^{\prime}\left(t^{(-1)}, t^{(-2)}\right)$ closes to order $\alpha$ if

$$
\Delta^{\prime}\left(Q_{-1}^{a}\right)=Q_{-1}^{a} \otimes 1+1 \otimes Q_{-1}^{a}+\alpha f^{a b c} Q_{-1}^{b} \otimes Q_{-1}^{c}+\ldots,
$$

where $Q_{-2}^{a} \equiv-f^{a b c} Q_{-1}^{b} Q_{-1}^{c}$, 目

The relations (3.53) imply that

$$
\left[Q_{0}^{a}, Q_{-1}^{b}\right]=f^{a b c} Q_{-1}^{c} .
$$

Other relations obtained from (3.53) are deformations of the affine Lie algebra ones. In particular, the Serre relations are deformed 0 . One finds

$$
\left[Q_{-2}^{a}, Q_{-1}^{b}\right]+\left[Q_{-2}^{b}, Q_{-1}^{a}\right]=\alpha^{2}\left(f^{a c d}\left\{Q_{-2}^{b}, Q_{-2}^{c}, Q_{-1}^{d}\right\}+f^{b c d}\left\{Q_{-2}^{a}, Q_{-2}^{c}, Q_{-1}^{d}\right\}\right)+\ldots
$$

4 The quantum determinant condition equal to one should also constrain the expansion (3.57), however we did not pursue this.

5 This corrects an error made in [10] [12]. 
The relation (3.60) is proved as follows. From (3.58) one can compute the comultiplication of the LHS of (3.60) to lowest order. This comultiplication is not consistent with the RHS equal to zero, but is consistent with the comultiplication of the RHS of (3.60) to lowest order.

Finally from the relations $(3.53)$ one may derive

$$
\left[Q_{1}^{a}, Q_{-1}^{b}\right]=f^{a b c} Q_{0}^{c}-\frac{\alpha^{2}}{4} f^{a b c}\left(Q_{-1}^{d} Q_{-1}^{d} Q_{0}^{c}+Q_{0}^{c} Q_{-1}^{d} Q_{-1}^{d}\right)+\ldots
$$

In the formulas (3.57)-(3.61) all of the corrections are infinite series in $\alpha$.

\subsection{Remarks on Form Factors}

Form factors are defined as matrix elements of fields on asymptotic states. For the field multiplets $\Phi_{\Lambda}(x)$, they are defined as

$$
f_{\Lambda}\left(\theta_{1}, . ., \theta_{n}\right)=\left\langle 0\left|\Phi_{\Lambda}(0) Z\left(\theta_{1}\right) \cdots Z\left(\theta_{n}\right)\right| 0\right\rangle
$$

The form factors may thus be understood as Clebsch-Gordon projectors for the above representations of the affine quantum group.

Smirnov explained how the fundamental axioms of the form factor bootstrap program may be understood as a consequence of the affine quantum group symmetry [12]. In this section we give a different derivation of the periodicity axiom ('deformed KnizhnikZamolodchikov equation'), in order to help clarify its meaning. The equations we derive improve slightly upon those obtained in [12] by making the data of the field module $\Lambda$ manifest. For completeness we consider the other axioms as well.

It is evident from the exchange relation (3.34) that $f_{\Lambda}$ has the following property upon exchange of two of its vector spaces:

$$
f_{\Lambda}\left(\theta_{1}, . ., \theta_{i}, \theta_{i+1}, . ., \theta_{n}\right)=S_{i, i+1}\left(\theta_{i, i+1}\right) f_{\Lambda}\left(\theta_{1}, . ., \theta_{i+1}, \theta_{i}, . ., \theta_{n}\right)
$$

The $2 \pi i$-periodicity axiom for the form factors may be derived by comparing form factors of the fields $\Phi_{\Lambda}$ and $\Phi_{\Lambda}^{\prime}$. From general QFT principles (mainly crossing symmetry) one finds that more general matrix elements can be obtained from the basic ones (3.62) as follows [15]:

$$
\left\langle\theta_{1}, . ., \theta_{m}\left|\Phi_{\Lambda}(0)\right| \theta_{m+1}, . ., \theta_{n}\right\rangle=f_{\Lambda}\left(\theta_{1}-i \pi, . ., \theta_{m}-i \pi, \theta_{m+1}, . ., \theta_{n}\right)
$$


(To simplify the presentation we are dropping transpositions of vector spaces and charge conjugation matrices in the above notation.) The field $\Phi_{\Lambda}^{\prime}(0)$ is related to $\Phi_{\Lambda}(0)$ by a rotation $\Phi_{\Lambda}^{\prime}(0)=R_{-\pi}\left(\Phi_{\Lambda}(0)\right)$. This rotation may be implemented by an operator $U_{-\pi}$ : $\Phi_{\Lambda}^{\prime}(0)=U_{-\pi}^{\dagger} \Phi_{\Lambda}(0) U_{-\pi}$. The operator $U_{-\pi}$ is antiunitarity due to the fact that it involves a time reversal. The form factors of $\Phi_{\Lambda}^{\prime}(0)$ are thus

$$
\begin{aligned}
\left\langle\theta_{1}, . ., \theta_{m}\left|\Phi_{\Lambda}^{\prime}(0)\right| \theta_{m+1}, . ., \theta_{n}\right\rangle & =\left\langle\theta_{1}, . ., \theta_{m}\left|U_{-\pi}^{\dagger} \Phi_{\Lambda}(0) U_{-\pi}\right| \theta_{m+1}, . ., \theta_{n}\right\rangle \\
& =\left\langle\theta_{m+1}+i \pi, . ., \theta_{n}+i \pi\left|\Phi_{\Lambda}(0)\right| \theta_{1}+i \pi, . ., \theta_{m}+i \pi\right\rangle \\
& =f_{\Lambda}\left(\theta_{m+1}, . ., \theta_{n}, \theta_{1}+i \pi, . ., \theta_{m}+i \pi\right)
\end{aligned}
$$

where we have used the antiunitarity of $U_{-\pi}$ and its action on the states. On the other hand, using the operator formulation of rotation (2.52) one finds

$$
\begin{aligned}
\left\langle\theta_{1}, . ., \theta_{m}\left|\Phi_{\Lambda}^{\prime}(0)\right| \theta_{m+1}, . ., \theta_{n}\right\rangle & =\left\langle\theta_{1}, . ., \theta_{m}\left|e^{a}\left(\Phi_{\Lambda}(0)\right) s^{\prime}\left(e_{a}\right)\right| \theta_{m+1}, . ., \theta_{n}\right\rangle \\
& =\mathcal{R}_{\rho_{V_{m+1} \cdot V_{n}}, \rho_{\Lambda}}^{-1} f_{\Lambda}\left(\theta_{1}-i \pi, . ., \theta_{m}-i \pi, \theta_{m+1}, . ., \theta_{n}\right) .
\end{aligned}
$$

The matrix $\mathcal{R}_{\rho_{V_{m+1} \cdots V_{n}}, \rho_{\Lambda}}$ is the universal $\mathcal{R}$-matrix evaluated in the indicated representations:

$$
\mathcal{R}_{\rho_{V_{m+1} \cdots V_{n}}, \rho_{\Lambda}}=\mathcal{R}_{\rho_{V_{n}}, \rho_{\Lambda}} \mathcal{R}_{\rho_{V_{n-1}}, \rho_{\Lambda}} \cdots \mathcal{R}_{\rho_{V_{m+1}}, \rho_{\Lambda}}
$$

Thus, comparing (3.65) and (3.66), one obtains

$$
f_{\Lambda}\left(\theta_{1}-2 \pi i, . ., \theta_{m}-2 \pi i, \theta_{m+1}, . ., \theta_{n}\right)=\mathcal{R}_{\rho_{V_{m+1} \cdots V_{n}}, \rho_{\Lambda}} f_{\Lambda}\left(\theta_{m+1}, . ., \theta_{n}, \theta_{1}, . ., \theta_{m}\right)
$$

To obtain a periodicity property, one can use (3.63) to exchange the vector spaces. For example for $m=1$ :

$$
f_{\Lambda}\left(\theta_{1}-2 \pi i, \theta_{2}, . ., \theta_{n}\right)=\mathcal{R}_{\rho_{V_{2} \cdot V_{n}}, \rho_{\Lambda}} S_{n 1} S_{n-1,1} \cdots S_{21} f_{\Lambda}\left(\theta_{1}, \theta_{2}, . ., \theta_{n}\right)
$$

The final form factor axiom may be derived from (3.39), by simply inserting this expression into (3.62) and using (3.50) 12]. As before, we give a version of this formula which displays the data of the module $\Lambda$ :

$$
\begin{aligned}
2 \pi i \underset{\theta_{i+1}=\theta_{i}-i \pi}{\operatorname{res}} & f_{\Lambda}\left(\theta_{1}, . ., \theta_{i}, \theta_{i+1}, . ., \theta_{n}\right) \\
= & {\left[\left(S_{i, i-1} S_{i, i-2} \cdots S_{i 1} \mathcal{R}_{V_{i}, \rho_{\Lambda}} S_{i, i+2} S_{i, i+3} \cdots S_{i n}\right)-1\right] } \\
& \cdot C^{a b}|a\rangle_{i} \otimes|b\rangle_{i+1} f_{\Lambda}\left(\theta_{1}, \theta_{2}, . ., \theta_{i-1}, \theta_{i+2}, . ., \theta_{n}\right) .
\end{aligned}
$$

When there is no bound state, the relations (3.63), (3.69) and (3.70) are all the form factor axioms. If there is bound states, an extra residue axiom should be added. 


\section{Discussion}

We conclude this paper with a discussion of some open problems. The relation (3.69) has been interpreted as a deformed Knizhnik-Zamolodchikov equation [13] [12]. A few remarks are in order. Consider the situation where the field is local, so that the factor $\mathcal{R}_{\rho_{V_{1} \cdot V_{n}}, \rho_{\Lambda}}$ is absent. Then our alternative derivation reveals that the relation (3.69) is actually only a consequence of elementary quantum field theory principles such as crossing symmetry. This is really no surprise given the fact that this property of form factors was proposed some time ago using bootstrap ideas. This suggests that this relation is a rather weak consequence of the affine quantum group symmetry, and that stronger constraints should exist.

The vertex operators $Z(\theta)$ were formally defined by their intertwining properties. It should be possible to construct an explicit representation of these operators in some auxiliary Hilbert space; e.g. a representation using auxiliary free fields similar to those used in the vertex operator representations of the quantum affine algebras [28]. Such a construction could lead to an efficient means of computing form factors.

The main difficulty in completely characterizing form factors from the affine quantum group symmetry is due to the fact that two very different kinds of representations are needed, the Verma module field representations $\rho_{\Lambda}$, and the finite dimensional representations $\rho_{V_{1} . V_{n}}$. The form factors provide an inner product for these two types of representations. One would like to establish a duality between the particle representations and the complete list of field representations, but it remains unclear whether this is possible. Such a result would imply that the full Hilbert space of the theory could be reconstructed from the action of the complete set of fields on the vacuum. This is actually the situation encountered in conformal field theory [29]. Certainly to obtain the complete set of fields will require descending with local integrals of motion in addition to the non-local ones. For massive theories such properties are generally very difficult to prove, since they rely on detailed knowledge of the operator product expansion, having a deep significance in the realm of axiomatic quantum field theory.

Finally, let us make an intriguing remark. It is well known that the classical integrable models are linearized once they are lifted in the classical double. In view of the fact that the $S$-matrix is given by the universal $\mathcal{R}$-matrix and that this $\mathcal{R}$-matrix is the canonical element, i.e. the identity, in $\mathcal{A} \otimes \mathcal{A}^{*}$ embedded in the double $\mathcal{D}(\mathcal{A}) \otimes \mathcal{D}(\mathcal{A})$, it is tempting to conjecture that integrable models admit free realizations once they are lifted into the quantum double. This idea may provide a starting point for constructing vertex operators. 


\section{Acknowledgements}

It is a pleasure to thank O. Babelon, G. Felder and F. Smirnov for discussions. This work is supported in part by NATO. A.L. is supported by the A. P. Sloan Foundation and the National Science Foundation. 


\section{Appendix A. Quantum Groups}

Let $\mathcal{A}$ denote a Hopf algebra. It is equipped with a multiplication map $m: \mathcal{A} \otimes \mathcal{A} \rightarrow \mathcal{A}$, a comultiplication $\Delta: \mathcal{A} \rightarrow \mathcal{A} \otimes \mathcal{A}$, antipode $s: \mathcal{A} \rightarrow \mathcal{A}$, and counit $\varepsilon: \mathcal{A} \rightarrow \mathbb{C}$. Let 1 denote the unit element, with $\Delta(1)=1 \otimes 1, s(1)=\epsilon(1)=1$. These operations have the following properties:

$$
\begin{aligned}
m(a \otimes 1) & =m(1 \otimes a)=a \\
m(m \otimes i d) & =m(i d \otimes m) \\
(\Delta \otimes i d) \Delta & =(i d \otimes \Delta) \Delta \\
\Delta(a) \Delta(b) & =\Delta(a b) \\
m(s \otimes i d) \Delta(a) & =m(i d \otimes s) \Delta(a)=\epsilon(a) \cdot 1 \\
s(a b) & =s(b) s(a) \\
\Delta s & =(s \otimes s) \Delta^{\prime} \\
(\epsilon \otimes i d) \Delta & =(i d \otimes \epsilon) \Delta=i d \\
\epsilon(a b) & =\epsilon(a) \epsilon(b),
\end{aligned}
$$

for $a, b \in \mathcal{A}$, and $\Delta^{\prime}$ is the skew comultiplication $\Delta^{\prime}=P \Delta$, where $P$ is the permutation operator $P(a \otimes b)=b \otimes a$. Eq. (A.1a) is the definition of the unit element, (A.1b, $)$ are the associativity and coassociativity of $\mathcal{A}$, (A.1d) defines $\Delta$ to be a homomorphism of $\mathcal{A}$ to $\mathcal{A} \otimes \mathcal{A}$, and $(\overline{\mathrm{A} .1 e-i})$ are the defining properties of the counit and antipode. The skew antipode $s^{\prime}$ is defined by the formula

$$
m\left(s^{\prime} \otimes i d\right) \Delta^{\prime}(a)=m\left(i d \otimes s^{\prime}\right) \Delta^{\prime}(a)=\epsilon(a) .
$$

Let $\left\{e_{a}\right\}$ denote a linear basis for $\mathcal{A}$. The above operations can be formulated by means of structure constants:

$$
\begin{aligned}
e_{a} e_{b} & =m_{a b}^{c} e_{c} \\
\Delta\left(e_{a}\right) & =\mu_{a}^{b c} e_{b} \otimes e_{c}, \quad \Delta^{\prime}\left(e_{a}\right)=\mu_{a}^{b c} e_{c} \otimes e_{b} \\
s\left(e_{a}\right) & =s_{a}^{b} e_{b} \\
\epsilon\left(e_{a}\right) & =\epsilon_{a}
\end{aligned}
$$

where $m_{a b}^{c}, \mu_{a}^{b c}, s_{a}^{b}$, and $\epsilon_{a}$ are constants in $\mathcal{C}$. We also define constants $\epsilon^{a}$ such that

$$
1=\epsilon^{a} e_{a}
$$


The properties (A.1) are easily expressed as consistency conditions on the structure constants:

$$
\begin{aligned}
m_{a b}^{c} \epsilon^{a} & =m_{b a}^{c} \epsilon^{a}=\delta_{b}^{c} \\
m_{a b}^{c} m_{d e}^{b} & =m_{a d}^{b} m_{b e}^{c} \\
\mu_{a}^{b c} \mu_{b}^{d e} & =\mu_{a}^{d b} \mu_{b}^{e c} \\
\mu_{a}^{i j} \mu_{b}^{k l} m_{i k}^{c} m_{j l}^{d} & =m_{a b}^{i} \mu_{i}^{c d} \\
m_{i j}^{b} s_{k}^{j} \mu_{a}^{i k} & =m_{j i}^{b} s_{k}^{j} \mu_{a}^{k i}=\epsilon_{a} \epsilon^{b} \\
m_{a b}^{i} s_{i}^{c} & =s_{b}^{j} s_{a}^{k} m_{j k}^{c} \\
\mu_{i}^{a b} s_{c}^{i} & =s_{j}^{a} s_{k}^{b} \mu_{c}^{k j} \\
\mu_{a}^{b c} \epsilon_{c} & =\mu_{a}^{c b} \epsilon_{c}=\delta_{a}^{b} \\
m_{a b}^{c} \epsilon_{c} & =\epsilon_{a} \epsilon_{b}
\end{aligned}
$$

The universal $\mathcal{R}$-matrix is a construction that will play an important role in the sequel. It is defined to satisfy the relation

$$
\mathcal{R} \Delta^{\prime}\left(e_{a}\right)=\Delta\left(e_{a}\right) \mathcal{R}
$$

for all $e_{a}$. The universal $\mathcal{R}$-matrix may be constructed formally by introducing the concept of the quantum double, due to Drinfeld. One defines a dual Hopf algebra $\mathcal{A}^{*}$ with linear basis $\left\{e^{a}\right\}$, and defines

$$
\mathcal{R}=\sum_{a} e_{a} \otimes e^{a} .
$$

The properties of $\mathcal{A}^{*}$ are fixed as follows. The relation (A.7) implies the following relations between the elements $\left\{e_{a}\right\}$ and $\left\{e^{a}\right\}$ :

$$
\mu_{a}^{b c} m_{b i}^{d} e_{c} e^{i}=\mu_{a}^{b c} m_{i c}^{d} e^{i} e_{b} .
$$

The multiplication map in $\mathcal{A}^{*}$ is fixed by requiring $\mathcal{R}$ to satisfy the Yang-Baxter equation:

$$
\mathcal{R}_{12} \mathcal{R}_{13} \mathcal{R}_{23}=\mathcal{R}_{23} \mathcal{R}_{13} \mathcal{R}_{12} .
$$

Inserting (A.7) into (A.9), one finds that the Yang-Baxter equation is satisfied if (A.8) holds along with

$$
e^{a} e^{b}=\mu_{c}^{b a} e^{c} .
$$


The comultiplication in $\mathcal{A}^{*}$ is fixed by imposing the quasi-triangularity condition

$$
(1 \otimes \Delta) \mathcal{R}_{12}=\mathcal{R}_{13} \mathcal{R}_{12}
$$

which implies

$$
\Delta\left(e^{a}\right)=m_{b c}^{a} e^{b} \otimes e^{c}
$$

Using the properties (A.5), one may define the antipode and counit such that $(\mathrm{A} .1 e-\mathrm{z})$ are satisfied in $\mathcal{A}^{*}$ :

$$
s\left(e^{a}\right)=\left(s^{\prime}\right)_{b}^{a} e^{b}, \quad \epsilon\left(e^{a}\right)=\epsilon^{a}, \quad \epsilon_{a} e^{a}=1 .
$$

One easily checks that the above definitions indeed endow $\mathcal{A}^{*}$ with a Hopf algebra structure by using the properties (A.5) of the structure constants. The Hopf algebra $\mathcal{A} \otimes \mathcal{A}^{*}$ is referred to as the quantum double $\mathcal{D}(\mathcal{A})$. One also has

$$
\mathcal{R}^{-1}=\sum_{a} s^{\prime}\left(e_{a}\right) \otimes e^{a}
$$

The relations (A.8) between elements of $\mathcal{A}$ and $\mathcal{A}^{*}$ can also be expressed in the form

$$
\begin{aligned}
e^{n} e_{m} & =\mu_{a}^{b c} \mu_{m}^{a f} m_{b i}^{d} m_{d j}^{n} s_{f}^{j} e_{c} e^{i} \\
e_{m} e^{n} & =\mu_{a}^{b c} m_{i c}^{d} \mu_{m}^{f a} m_{j d}^{n} s_{f}^{j} e^{i} e_{b} .
\end{aligned}
$$

The formula (A.15a) is established by multiplying both sides of (A.8) by $\mu_{m}^{a f} m_{d j}^{n} s_{f}^{j}$ and using (A.5b,c,e,a,h) . Equation (A.15b) is similarly proven.

\section{Appendix B. Verifying the Current Representation of $\mathcal{D}(\mathcal{A})$.}

In this appendix we prove that the current representation $\rho_{J}$ defined in (2.67) and (2.71) satisfy the defining relations of $\mathcal{D}(\mathcal{A})$.

By construction, namely due to the property (2.26) of the adjoint action, $\rho_{J}$ satisfies the multiplication law in $\mathcal{A}$ :

$$
\rho_{J}\left(e_{a}\right)_{i}^{d} \rho_{J}\left(e_{b}\right)_{c}^{i}=m_{a b}^{j} \rho_{J}\left(e_{j}\right)_{c}^{d}
$$

The coassociativity condition (A.5d) ensures that $\rho_{J}\left(e^{a}\right)$ satisfies the multiplication law in $\mathcal{A}^{*}$ :

$$
\rho_{J}\left(e^{a}\right)_{i}^{d} \rho_{J}\left(e^{b}\right)_{c}^{i}=\mu_{i}^{b a} \rho_{J}\left(e^{i}\right)_{c}^{d}
$$


That the current representation also satisfies the defining relation (2.49) between elements of $\mathcal{A}$ and $\mathcal{A}^{*}$ is more non-trivial, and may be proven as follows. Inserting the current representation into (2.49), one finds these latter relations are satisfied if

$$
\mu_{a}^{b c} m_{b i}^{d} \mu_{m}^{i l} \widetilde{f}_{c l}^{n}=\mu_{a}^{b c} m_{i c}^{d} \widetilde{f}_{b m}^{l} \mu_{l}^{i n}
$$

An indirect proof of the identity $(\overline{B .3})$ can be given as follows. The $\Delta$-homomorphism property (A.1d) applied to the relation (2.29), namely, requiring $\Delta\left(Q_{a}\right)$ to also satisfy (2.29), leads to the formula

$$
Q_{a} \Theta_{b}^{c}+f_{i j}^{c} \Theta_{a}^{i} \Theta_{b}^{j}=f_{a b}^{d} \Theta_{d}^{c}+R_{a b}^{e d} \Theta_{d}^{c} Q_{e} .
$$

Evaluating (B.4) in the adjoint representation(2.27) by using (2.23) and (2.30) yields directly the identity (B.3). 


\section{Figure Captions}

Fig. 1. Graphical representation of non-local currents.

Fig. 2. Action of $\Theta_{a}^{b}$ on a field.

Fig. 3. Contour decomposition of the adjoint action.

Fig. 4. Contour decomposition leading to the comultiplication.

Fig. 5. $\quad R_{\pi}^{2}$ on a non-local current.

Fig. 6. Euclidean rotation used to compute the antipodes.

Fig. 7. $a d^{\prime}$ action.

Fig. 8. Graphical representation of eq. (2.75). 


\section{References}

[1] E. K. Sklyanin, L. A. Takhtadzhyan, and L. D. Faddeev, Theor. Math. 40 (1980) 688.

[2] L. Faddeev, Les Houches Lectures 1982, Elsevier Science Publishers (1984).

[3] D. Bernard and A. LeClair, Phys. Lett. B247 (1990) 309.

[4] D. Bernard and A. LeClair, Commun. Math. Phys. 142 (1991) 99.

[5] G. Felder and A. LeClair, 'Restricted Quantum Affine Symmetry of Perturbed Minimal Conformal Models', RIMS preprint 799 and Cornell preprint CLNS 91/1099, to appear in Int. J. Mod. Phys.

[6] M. Lüscher, Nucl. Phys. B135 (1978) 1.

[7] D. Bernard, Commun. Math. Phys. 137 (1991) 191.

[8] A. Zamolodchikov, Landau Institute preprint, Sept. 1989, unpublished.

[9] A. B. Zamolodchikov and Al. B. Zamolodchikov, Annals Phys. 120 (1979) 253.

[10] A. LeClair and F. A. Smirnov, Int. J. Mod. Phys. A7 (1992) 2997.

[11] D. Bernard and G. Felder, Nucl. Phys. B365 (1991) 98.

[12] F. A. Smirnov, 'Dynamical Symmetries of Massive Integrable Models', RIMS preprint 772,838 (1991).

[13] I. B. Frenkel and N. Yu. Reshetikhin, proceedings of XX-th International Conference on Differential Geometric Methods in Theoretical Physics, eds. S. Catto and A. Rocha, World Scientific (1992).

[14] B. Davies, O. Foda, M. Jimbo, T. Miwa, and A. Nakayashiki, 'Diagonalization of the XXZ Hamiltonian by Vertex Operators', RIMS preprint (1992).

[15] F. A. Smirnov, Form Factors in Completely Integrable Models of Quantum Field Theory, Advanced Series in Mathematical Physics 14, World Scientific, 1992;

A. N. Kirillov and F. A. Smirnov, Phys. Lett. 198B (1987) 506; Zap. Nauch. Semin. LOMI 164 (1987) 80.

[16] C. Gómez and G. Sierra, Nucl. Phys. B 352 (1991) 791.

[17] G. Felder and C. Wieczerkowski, Commun. Math. Phys. 131 (1990) 125.

[18] V.G. Knizhnik and A.B. Zamolodchikov, Nucl. Phys. B297 (1984) 83.

[19] M. B. Halpern, Phys. Rev. D12 (1976) 1684.

[20] A. B. and Al. B. Zamolodchikov, Phys. Lett. 72B (1978) 481.

[21] V. G. Drinfel'd, Sov. Math. Dokl. 32 (1985) 254; Sov. Math. Dokl. 36 (1988) 212; 'Quantum Groups', Proceedings of the International Congress of Mathematicians, Berkeley, CA, 1986.

[22] N. Yu. Reshetikhin and M. A. Semenov-Tian-Shansky, Lett. Math. Phys. 19 (1990) 133.

[23] M. Jimbo, Lett. Math. Phys. 10 (1985) 63; Lett. Math. Phys. 11 (1986) 247; Commun. Math. Phys. 102 (1986) 537; Int. J. Mod. Phys. A4 (1989) 3759. 
[24] L. D. Faddeev, N. Yu. Reshetikhin, and L. A. Takhtajan, Algebra and Analysis 1 (1989) 178.

[25] V. G. Drinfel'd, Leningrad Math. J. 1 (1990) 1419

[26] P. P. Kulish and E. K. Sklyanin, J. Soviet Math. 19, p61.

[27] A.Yu. Alekseev and L.D. Faddeev, Commun. Math. Phys. 141 (1991) 413

[28] I. B. Frenkel and N. H. Jing, Proc. Nat'l. Acad. Sci. (USA) 85 (1988) 9373.

[29] A. A. Belavin, A. M. Polyakov, and A. B. Zamolodchikov, Nucl. Phys. B241 (1984) 333. 